\title{
Has the Pandemic Triggered a 'Paperdemic'? Towards an Assessment of Diagnostic Indicators for COVID-19
}

\author{
Ali Muhammad Ali Rushdi ${ }^{{ }^{*}}$ and Hamzah Abdul Majid Serag ${ }^{1}$ \\ ${ }^{1}$ Department of Electrical and Computer Engineering, King Abdulaziz University, P.O.Box 80200, \\ Jeddah 21589, Saudi Arabia.
}

Authors' contributions

This work was carried out in collaboration between the two authors. Author AMAR wrote the entire draft of the manuscript, conducted the mathematical and conceptual analyses and managed the basic literature survey. Author HAMS participated in the literature search, performed the computational work and constructed the table of results. Both authors read and approved the final manuscript.

Article Information

DOI: $10.9734 / / J P R / 2021 / v 6 i 230158$ Editor(s):

(1) Dr. Khadiga Ahmed Ismail Eltris, Ain Shams University, Egypt. (2) Prof. John Yahya I. Elshimali, UCLA School of Medicine, Charles R. Drew University of Medicine and Science, USA.

(3) Dr. Rafik Karaman, Al-Quds University, Palestine. Reviewers:

(1) Muneeb Ahmad, Jiangxi University of Finance and Economics, China.

(2) Mengistu Hailemariam, Hawassa University, Ethiopia.

(3) Lucia Azocar Aedo, Universidad San Sebastián, Chile. (4) Marcel Vasconcellos, Centro Universitário Serra dos Órgãos (UNIFESO), Brazil. Complete Peer review History: http://www.sdiarticle4.com/review-history/65260

Review Article

Received 18 January 2021

Accepted 22 February 2021

Published 01 March 2021

\section{ABSTRACT}

This paper is a preliminary step towards the assessment of an alarming widespread belief that victims of the novel coronavirus SARS-CoV-2 include the quality and accuracy of scientific publications about it. Our initial results suggest that this belief cannot be readily ignored, denied, dismissed or refuted, since some genuine supporting evidence can be forwarded for it. This evidence includes an obvious increase in retractions of papers published about the COVID-19 pandemic plus an extra-ordinary phenomenon of inconsistency that we report herein. In fact, we provide a novel method for validating any purported set of the four most prominent indicators of diagnostic testing (Sensitivity, Specificity, Positive Predictive Value, and Negative Predictive Value), by observing that these indicators constitute three rather than four independent quantities. This observation has virtually been unheard of in the 
open medical literature, and hence researchers have not taken it into consideration. We define two functions, which serve as consistency criteria, since each of them checks consistency for any set of four numerical values (naturally belonging to the interval $[0.0,1.0]$ ) claimed to be the four basic diagnostic indicators. Most of the data we came across in various international journals met our criteria for consistency, but in a few cases, there were obvious unexplained blunders. We explored the same consistency problem for some diagnostic data published in 2020 concerning the ongoing COVID-19 pandemic and observed that the afore-mentioned unexplained blunders tended to be on the rise. A systematic extensive statistical assessment of this presumed tendency is warranted.

Keywords: COVID-19; compromised standards; diagnostic testing; sensitivity; specificity; positive predictive value; negative predictive value; consistency criterion.

\section{INTRODUCTION}

The world currently witnesses an ongoing epidemic of the novel coronavirus (SARS-CoV-2) that causes the disease COVID-19, now characterized as a pandemic by the World Health Organization (WHO) [1-6]. This pandemic seems to have expanded from the Wuhan province in China, but has definitely reached (in repetitive waves) almost every inhabited territory on the globe. This fatal disease, being a catastrophic threat of dramatic public-health and economic concerns proved to have diverse grave (and potentially irreversible) consequences [1-4]. For the past thirty years, a once-per-decade novel coronavirus has pushed the global public health system to the limit, with SARS-CoV-2 being the most severe. Despite repeated warnings, the world was not prepared for that pandemic [7], and the world's response to the pandemic leaves a lot to be desired. Now, there is a genuine need for further research concerning various aspects of epidemiology, in general, with a stress on pathogen research related to COVID-19, in particular. This research might pay off handsomely in improving the response of humanity to this pandemic. The past year (2020) witnessed a dramatic increase in the number of published scientific papers supposedly reporting results of the required research. There is a great concern that publication standards have been compromised as the peer-review process is becoming hasty and weak [8-16]. There are suspicions that scientific publications concerning COVID-19 are ranging from robust and rigorous studies to dishonest, incompetent, or fraudulent studies being conducted, posted, and shared at an unprecedented rate. Dinis-Oliveira [8] coined the term 'paperdemic' to refer to a parallel virtual viral pandemic (ignited by the genuine pathogenic pandemic) that leads to publications based on mistakes or misconduct. He asserts that A pandemic with a "paperdemic" will be even more complicated to manage if it progresses in an uncontrolled manner and is not properly scrutinized.

The role of gatekeepers of science is the collective responsibility of scientists in general [17]. It is also an implicit assignment or task for the contemporary system of scientific publishing. This system addresses such a task in a proactive strategy (the peer-review process) supplemented by a reactive manner (paper retraction schemes). Since we are suspicious of the proactive strategy in the COVID-19 era [18-20], we will look now at the reactive paradigm to see if it supports the hypothesized phenomenon of compromised standards and if it can serve to mitigate it. Paper retraction might only address severe issues such as fraud (author engagement in research misconduct or author use of deliberately flawed, fabricated, falsified or fraudulent data) or errors (plagiarism or scientific mistakes). Retractions are in some cases accompanied by explanations and apologies for what warrants the total content withdrawal (rather than a limited-scope correction). A decade ago, Steen [21] observed that the recent rate of increase in retractions was generally greater than the corresponding rate of increase in publications. Moreover, he noted that in the 6 years between 2004 and 2009, the increase in the number of papers retracted for fraud was much more than that retracted for scientific mistakes [21]. Vuong [22] asserts that retractions are not intrinsically bad, as they serve as a practical way to correct for human fallibility and to keep the scientific literature trustworthy. He points out that the scientific community should agree on the essential information to be provided when pulling a paper from the scientific literature. Definitely, retraction notices should be more informative [22,23]. During the COVID-19 pandemic, an exceptionally fast track became available for the peer review of papers dealing with the pandemic. The availability of such a fast track is blamed for further increase in the number of papers that have been retracted due to quality 
and/or data issues [22,24-26]. In fact, such a fast track is widely accused of being of low-quality despite vigorous denial by publishers.

Even before the COVID-19 era, there have been many methods (mainly statistical) for detecting false data [27-37], that have become rather visible and advanced during the past decade. Rushdi and Rushdi [38,39] have recently suggested methods for avoiding probabilistic fallacies in medical context. In [40], they introduced a non-statistical method based on the premise that flawed data might be detected via the excessive inconsistencies it causes in a variant of Boolean Analysis called Qualitative Comparative Analysis (QCA) [40-44]. Rushdi and Serag $[4,45]$ developed yet another nonstatistical checking method, which can be used for validating a certain category of bio-statistical data. This method is based on a newlydiscovered inter-relation among the four most prominent indicators of diagnostic testing (sensitivity, specificity, positive predictive value (PPV), and negative predictive value (NPV)) [4,40,45-49]. Rushdi and Serag [4,45] developed simple formulas that express any one of these four indicators in terms of the other three. They called a set of four values satisfying these formulas (to within permissible round-off errors) a consistent set. Extensive testing was made for sets of the four basic indicators published recently in a variety of international medical journals [45], and in various Saudi medical journals [17], to check whether these sets are consistent or not. Most of the data they came across met their criteria for consistency, but in a few cases, there were obvious unexplained blunders. The present paper aims to explore the same consistency problem for some diagnostic data published in 2020 concerning the ongoing COVID-19 pandemic. We observe that the aforementioned unexplained blunders tended to be on the rise within publications about COVID-19, and we suggest that a systematic extensive statistical assessment of this presumed tendency is warranted.

The organization of the rest of this paper is as follows. Section 2 is a standard section of materials and methods. It starts with a brief primer about diagnostic testing and its basic measures. Section 2 also presents the essential machinery on which this paper is based. It reports virtually unknown formulas for interdependence among the two predictive values, sensitivity, and specificity. These formulas express any one of these four indicators in terms of the other three, under the assumption that each of the four exists, and no division by zero is encountered. Section 3 reports our computational results, obtained by applying the new formulas extensively to some data published in 2020 about diagnostic measures for COVID19. Most sets of values of sensitivity, specificity, and predictive values tested agree with our formulas, thereby independently attesting to the correctness of these formulas. However, some reported sets of the four basic indicators experience some appreciable incoherence among their values according to our formulas. The percentage of incoherent cases seems definitely higher than it was in $[17,45]$. Section 4 presents a detailed discussion of the importance of our findings, while Section 5 concludes the paper.

\section{MATERIALS AND METHODS}

\subsection{On Diagnostic Testing and Its Basic Measures}

This subsection is intended for a brief primer about diagnostic testing and its most basic indicators [38-40,45-49]. Fig. 1 demonstrates a two-by-two contingency matrix for test or classification $i$ with respect to test or classification $j$. Each of the two variables $i$ and $j$ is a dichotomous variable that belongs to the set $\{+1,-1\}$ of indices. The test $i$ reports 'positive' cases (arbitrarily assigned the value +1 ), in which a certain disease, attribute, trait, or condition is present, or reports 'negative' cases (arbitrarily assigned the value -1 ), in which this disease, attribute, trait, or condition is absent. This test is assessed or evaluated by a reference or gold standard test $j$, which has its own labeling of cases, again as positive or negative. The reference test $j$ designates various cases of the assessed test $i$ as "true" or "false," depending on whether it agrees or disagrees with test $i$, respectively. As a result, the matrix four entries are called True Positives, False Positives, False Negatives, and True Negatives. These entries are usually assigned the standard abbreviations $T P, F P, F N$, and $T N$. In the sequel, we will use the subscripted abbreviations $T P_{i j}, F P_{i j}, F N_{i j}$, and $T N_{i j}$, where we use the subscripts $i j$ for all measures (and later for indicators derived from them) to assert the notion that $i$ is assessed, judged or measured relative to $j$. The sum of these four entries is the size of the reported population or the total number of reported cases $\mathrm{N}$. If the tests $i$ and $j$ interchange their roles (so that test $j$ is now assessed lative 
to test $i$ ) then the four measures are relabeled as $T P_{j i}, F P_{j i}, F N_{j i}$, and $T N_{j i}$ such that $T P_{j i}=T P_{i j}$, and $T N_{j i}=T N_{i j}$ but with $F P_{j i}=F N_{i j}$, and $F N_{j i}=F P_{i j}$. This is the reason why omission of the subscripts is not desirable, as it leads to an inadvertent ambiguity as to which assesses which.

We use the symbols $A=\{j=+1\}$ and $B=\{i=$ $+1\}$ to denote the events of positive cases (presence of the considered condition) according to the tests $j$ and $i$, respectively. Hence, the complementary events $\bar{A}=\{j=-1\}$ and $\bar{B}=\{i=$ -1 ) denote the events of negative cases (absence of the considered condition) according to the tests $j$ and $i$, respectively. There are eight conditional probabilities concerning these two events and their complements, as shown in Fig. 2. These can be identified as the eight most prominent indicators used in diagnostic testing. These are the Sensitivity $\left(\operatorname{Sens}_{i j}\right)$ or True Positive Rate $\left(T P R_{i j}\right)$, the Specificity $\left(\operatorname{Spec}_{i j}\right)$ or True Negative Rate $\left(T N R_{i j}\right)$, the Positive and Negative Predictive Values $\left(P P V_{i j}\right.$ and $\left.N P V_{i j}\right)$, together with their respective complements (to 1.0), namely the False Negative Rate $\left(F N R_{i j}\right)$, False Positive Rate $\left(F P R_{i j}\right)$, False Discovery rate $\left(F D R_{i j}\right)$ and False Omission Rate $\left(F O R_{i j}\right)$ [3840,45-49]. The former four indicators are considered more popular or more prominent, and they act as direct or agreement measures the latter four serve as discrepancy or disagreement measures between the two tests $i$ and $j$. Due to the four complementation relations within pairs of these eight measures, the number of independent quantities among them is at most four. It seems that there is a widespread (and at least implicit) belief that this number is exactly four (usually obtained by counting the four direct indicators $\operatorname{Sens}_{i j}, \operatorname{Spec}_{i j}, P P V_{i j}$ and $N P V_{i j}$ ). We show in Section 3 that this number is, in fact, three, by simply being able to express any of the four direct indicators in terms of the other three.

Note that each conditional probability in Fig. 2 has a 'dual' one obtained by complementing both the conditioned and conditioning events [50], and also has an inverse or transposed one, obtained by swapping or interchanging the conditioned and conditioning events $[38,39]$. Our definition of 'duality' is in line with that used with Boolean quantities, where duality is achieved through complementing both the input and output quantities [51-53]. Our definitions of duality and transposition mean that each conditional probability $P$ has a dual $P^{d}$, a transpose or inverse $T$, and a dual of its transpose or inverse (a transpose of its dual) $T^{d}$. Note that both the duality and transposition operators are involutary or self-inverse operators, i.e., each of them satisfies 'the law of involution' (applying any of them twice to a specific conditional probability leaves it intact) [54-56]. Table 1 defines the four possible sets $\left\{P, P^{d}, T, T^{d}\right\}$ pertaining to the set of four direct indicators of diagnostic testing. Similar definitions apply to the set of complementary indicators of diagnostic testing. Fig. 3 is yet another geometric display of the inter-relationships among the eight diagnostic indicators defined in Fig. 2. Two conditional probabilities constituting a dual pair are placed on the same vertical line, while two conditional probabilities constituting a transpose or inverse pair are situated on the same horizontal line. Hence, any conditional probability and the dual of its transpose or inverse (the transpose of its dual) appear diagrammatically opposite. In Fig. 3 , the set of four direct indicators of diagnostic testing is distinguished in blue, while the set of complementary indicators of diagnostic testing is highlighted in red. Each member in the first set has a one-to-one and onto mapping to a member in the second set, which is its complement (to one).

\subsection{Validating Formulas Used in the Analysis}

We now express each of the four most prominent indicators of diagnostic testing (Specificity, Negative Predictive Value, Sensitivity, and Positive Predictive Value) solely in terms of the other three (provided each of the four indicators exists, and no division by zero is encountered), namely $[4,17,45]$.

$$
\begin{aligned}
& \operatorname{Sens}_{i j} \\
& =\frac{P P V_{i j} * N P V_{i j}\left[1-\operatorname{Spec}_{i j}\right]}{P P V_{i j} N P V_{i j}+\operatorname{Spec}_{i j}\left[1-P P V_{i j}-N P V_{i j}\right]}
\end{aligned}
$$

$$
\begin{aligned}
& \operatorname{Spec}_{i j} \\
& =\frac{P P V_{i j} * N P V_{i j}\left[1-\operatorname{Sens}_{i j}\right]}{P P V_{i j} * N P V_{i j}+\operatorname{Sens}_{i j}\left[1-P P V_{i j}-N P V_{i j}\right]} \\
& P P V_{i j} \\
& =\frac{\operatorname{Sens}_{i j} * \operatorname{Spec}_{i j}\left[1-N P V_{i j}\right]}{\operatorname{Sens}_{i j} * \operatorname{Spec}_{i j}+N P V_{i j}\left[1-\operatorname{Sens}_{i j}-\operatorname{Spec}_{i j}\right]}
\end{aligned}
$$




$$
\begin{aligned}
& N P V_{i j} \\
& =\frac{\operatorname{Sens}_{i j} * \operatorname{Spec}_{i j}\left[1-P P V_{i j}\right]}{\operatorname{Sens}_{i j} * \operatorname{Spec}_{i j}+P P V_{i j}\left[1-\operatorname{Sens}_{i j}-\operatorname{Spec}_{i j}\right]}
\end{aligned}
$$

Equations (1-4) might be written in a unified form (See Table 1) as

$$
P=\frac{T * T^{d}\left[1-P^{d}\right]}{T * T^{d}+P^{d}\left[1-T-T^{d}\right]}
$$

We also define two checking functions of these four values that we call the Diagnostic Checking Difference (DCD) and the Diagnostic Checking Ratio (DCR), that are exactly 0 and 1 , respectively, for consistent values. The mathematical definition of the DCD and DCR is [4].

$$
\begin{aligned}
& D C D_{i j}=\operatorname{Sens}_{i j} * \operatorname{Spec}_{i j}\left[P P V_{i j}+N P V_{i j}-\right. \\
& 1-P P V i j * N P V i j\left[\text {Sensij}_{\text {SPecij-1 }}\right. \text { (6) } \\
& D C R_{i j} \\
& =\frac{\operatorname{Sens}_{i j} * \operatorname{Spec}_{i j}\left[P P V_{i j}+N P V_{i j}-1\right]}{P P V_{i j} * * N P V_{i j}\left[\text { Sens }_{i j}+\text { Spec }_{i j}-1\right]}
\end{aligned}
$$

We reiterate that we use the subscripts $i j$ for all measures and indicators to assert the notion that test $i$ is assessed, judged or measured relative to the reference test or gold standard $j$. Equations (6) and (7) might be written in a generalized form (See Table 1) as.

$$
\begin{aligned}
& +D C D_{i j} \text { OR }-D C D_{i j}=P * P^{d}\left[T+T^{d}-1\right]- \\
& T * T^{d}\left[P+P^{d}-1\right] . \\
& D C R_{i j} \text { OR }\left(1 / D C R_{i j}\right) \\
& =\frac{P * P^{d}\left[T+T^{d}-1\right]}{T * T^{d}\left[P+P^{d}-1\right]}
\end{aligned}
$$

The fact that the expressions in (8) and (9) are identically equal to 0 and 1 , respectively, means that the quantity $\left(P * P^{d}\left[T+T^{d}-1\right]\right)$, which is naturally invariant to the replacement of every term by its dual, is also invariant to the replacement of every term by its transpose.

\section{RESULTS}

This section reports our results, followed by an assessment of some (arbitrary selected) diagnostic data reported in the COVID-19 Era. We note that the deviation of the DCD and the DCR from 0 and 1 , respectively, is a measure of inconsistency for any purported set of the four diagnostic indicators (sensitivity, specificity, positive predictive value (PPV), and negative predictive value (NPV)). Since members of such a purported set are (conditional) probabilities, they are expected to be non-negative fractional vales belonging to the interval $[0.0,1.0]$. Table 2 provides our validation of some published sets of these four basic indicators within some publications that appeared in 2020 on the COVID-19 pandemic. We check whether the sets considered are consistent or not. For each published set of $\left\{\operatorname{Sens}_{i j}, \operatorname{Spec}_{i j}, P P V_{i j}, N P V_{i j}\right\}$ the table computes the checking difference $D C D_{i j}$ via (6), and the checking ratio $D C R_{i j}$ via (7). It also uses equations (1-4) to compute a new value for each of the four prominent indicators in terms of the old values of the other three indicators. We arbitrarily assume that a published set is consistent (uncolored entries) if the absolute value of the relative error is less than or equal to $2 \%$. We arbitrarily consider such a small error accountable for by normal or acceptable round-off errors [5762]. Otherwise, we consider a set to be somewhat problematic (with error still within $4 \%$, highlighted in yellow), or inconsistent (with error still within 6\%, highlighted in orange). If the absolute relative error exceeds $6 \%$, we arbitrarily label the corresponding set as dramatically inconsistent (highlighted in red). Most sets of values of sensitivity, specificity, and predictive values tested agree with our formulas, thereby independently attesting to the correctness of these formulas. However, some reported sets of the four basic indicators experience some appreciable incoherence among their values according to our formulas. Similar results were earlier obtained in [45] for data randomly selected from international journals and in [17] for data randomly selected from Saudi medical journals. The percentage of incoherent cases and the severity of their inconsistency seem definitely higher for the present COVID-19 data than they were in $[17,45]$. In fact, less than half of the articles covered in Table 2 are totally free of any inconsistency problem.

\section{DISCUSSION}

Our results could become a useful future methodology to verify a certain aspect of the quality of medical articles. This methodology would complement other existing guidelines for the reporting of medical research [63-71]. The purpose of having these guidelines is to establish a recipe or "manual for the authors to follow, which should lead to total transparency, accurate 
reporting, and easier assessment of the validity of reported research findings [69]." The six most widely accepted and used guidelines are: PRISMA, CONSORT, STROBE, MOOSE, STARD, and SPIRIT. Johansen and Thomsen [69] assert that the implementation of these guidelines has led to only a moderate improvement in the quality of the reporting of medical research, and that there is still much work to be done to achieve accurate and transparent reporting of medical research findings. We hope the validating formulas reported herein would receive the popularization they deserve, and that future medical publications would take them into consideration. We do not anticipate a dramatic improvement in the quality of reporting of medical research, but we hope that our humble contribution would serve as a modest step of a process of continuous improvement. Moher and Altman [67] assert that "making a major impact on the quality of reporting and mitigating deficiencies is a huge challenge because no one group has prime responsibility and no single action is likely to have a large impact." We agree with the four proposals they made to help improve the medical research literature, namely: "(1) introducing publications officers; (2) developing core competencies for editors and peer reviewers, around which (3) training can be tailored; and (4) training authors to write articles fit for purpose."

\begin{tabular}{|c|c|c|}
\hline$i$ & +1 & -1 \\
\hline+1 & $\begin{array}{c}T P_{i j} \\
\text { (True Positives) }\end{array}$ & $\begin{array}{c}F_{i j} \\
\text { (False Positives) } \\
\text { (Type I Error) }\end{array}$ \\
\hline-1 & $\begin{array}{c}\text { (False Negatives) } \\
\text { (Type II Error) }\end{array}$ & $\begin{array}{c}T N_{i j} \\
\text { (True Negatives) }\end{array}$ \\
\hline
\end{tabular}

Fig. 1. The two-by-two contingency matrix of test or classification $i$ with respect to test or classification $j$. This matrix has integer entries that add to the total number of cases $N$. The symbols $A=\{j=+1\}$ and $B=\{i=+1\}$ denote the events of positive cases according to tests $j$ and $i$, respectively

Table 1. Possible definitions of a conditional probability $P$, its dual $P^{d}$, its transpose or inverse $T$, and the dual of its transpose or inverse (transpose of its dual) $T^{d}$. These definitions pertain to the set of four direct indicators of diagnostic testing. Similar definitions apply to the set of complementary indicators of diagnostic testing

\begin{tabular}{|c|c|c|c|}
\hline $\mathbf{P}$ & $\mathbf{P}^{\mathbf{d}}$ & $\mathbf{T}$ & $T^{d}$ \\
\hline Sens $_{i j}$ & Spec $_{i j}$ & $P P V_{i j}$ & $N P V_{i j}$ \\
\hline Spec $_{i j}$ & Sens $_{i j}$ & $N P V_{i j}$ & $P P V_{i j}$ \\
\hline$P P V_{i j}$ & $N P V_{i j}$ & Sens $_{i j}$ & Spec $_{i j}$ \\
\hline$N P V_{i j}$ & $P P V_{i j}$ & Spec $_{i j}$ & Sens $_{i j}$ \\
\hline
\end{tabular}




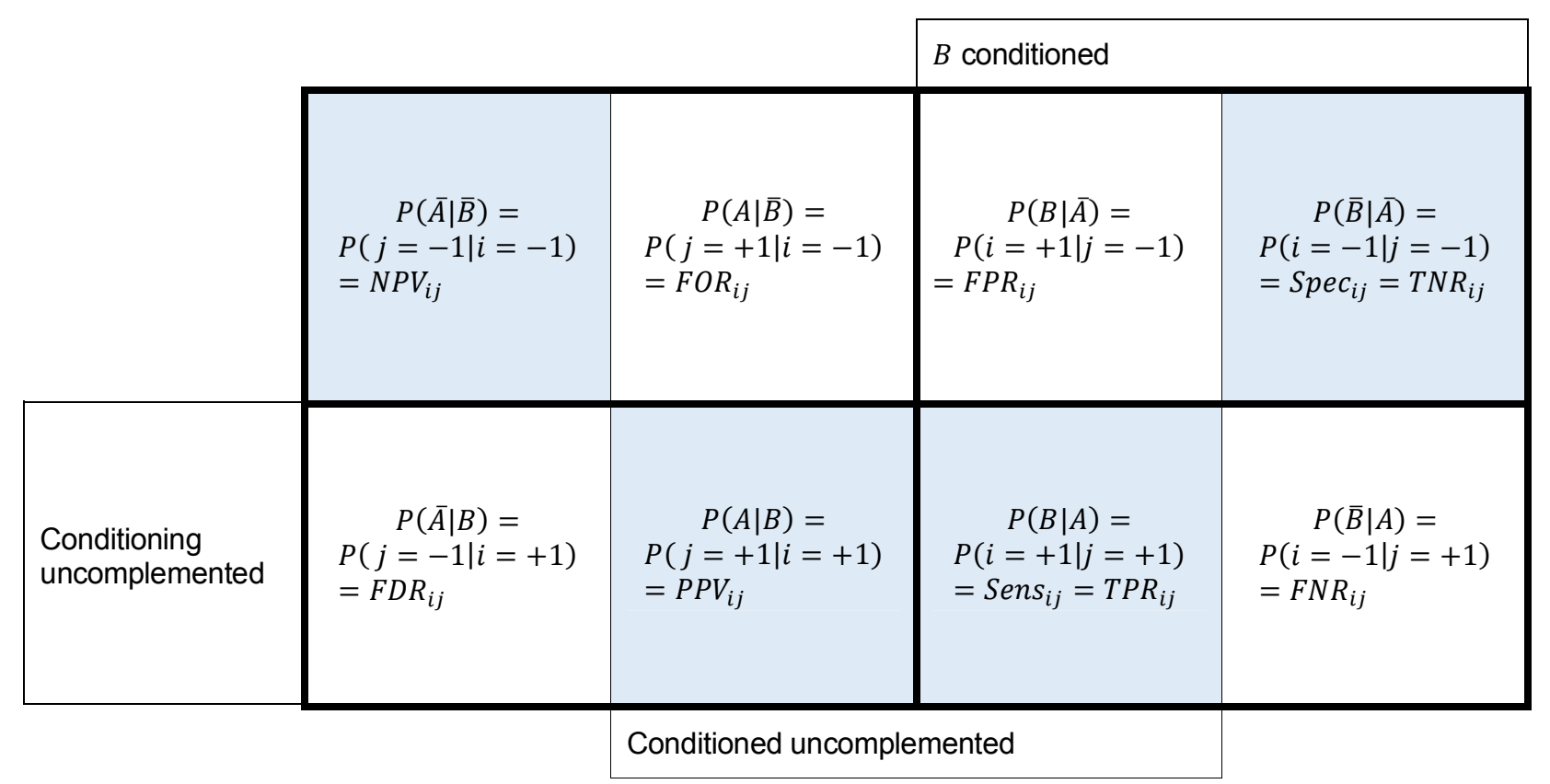

Fig. 2. Definition of the eight conditional probabilities concerning events $A=\{j=+1\}$ and $B=\{i=+1\}$, which constitute the eight most prominent indicators of diagnostic testing. The four shaded entries are direct indicators, usually taken for the most basic ones. The four unshaded entries are complementary indicators. Each conditional probability has a 'dual' one obtained by complementing both the conditioned and conditioning events, and also has an inverse or transposed one, obtained by swapping the conditioned and conditioning events 


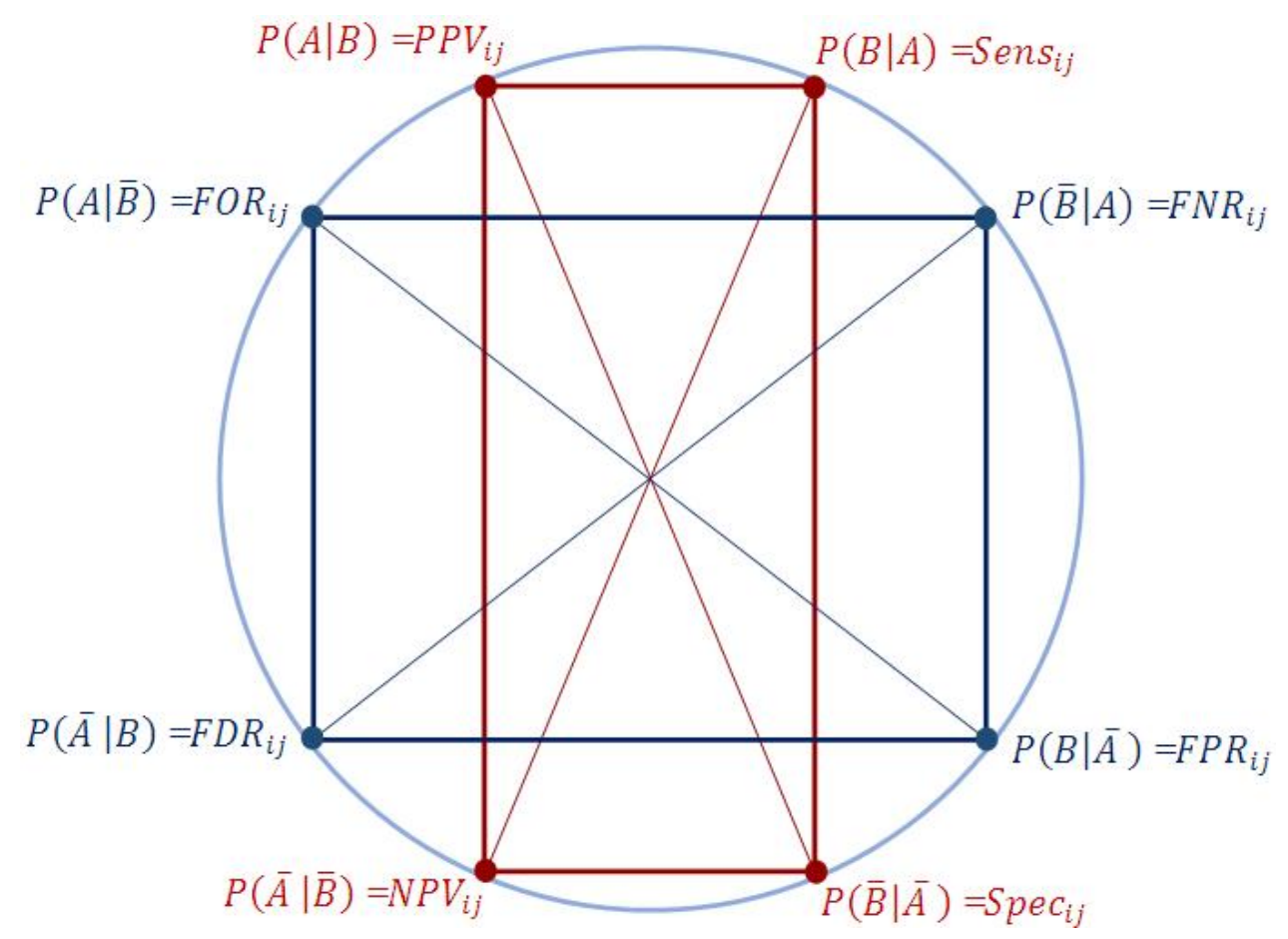

Fig. 3. Geometric display of the inter-relationships among the eight diagnostic indicators defined in Fig. 2. Each of these indicators is a conditional probability $P$ that has a dual $P^{d}$ (on the same vertical line), a transpose or inverse $T$ (on the same horizontal line), and a dual of its transpose or inverse (a transpose of its dual) $T^{d}$ (diagrammatically opposite). The set of four direct indicators of diagnostic testing is distinguished in blue,

while the set of complementary indicators of diagnostic testing is highlighted in red 
Table 2. Checking consistency among sets of the four prominent diagnostic indicators published in 2020 on topics related to the Covid-19 pandemic. In a dominant majority of cases, the published sets are consistent (uncolored entries), and in a small number of cases, there are sets that are somewhat problematic (highlighted in yellow), or dramatically inconsistent (highlighted in red)

\begin{tabular}{|c|c|c|c|c|c|c|c|c|c|c|c|c|}
\hline \multirow[t]{2}{*}{ \# } & \multicolumn{4}{|c|}{ Original values } & \multicolumn{2}{|c|}{$\begin{array}{c}\text { Checking } \\
\text { Values }\end{array}$} & \multicolumn{4}{|c|}{ Computed values } & \multirow[t]{2}{*}{ Source } & \multirow{2}{*}{$\begin{array}{l}\text { Data } \\
\text { location } \\
\text { in } \\
\text { Source }\end{array}$} \\
\hline & Sens $_{i j}$ & Spec $_{i j}$ & $\mathbf{P P V}_{\mathrm{ij}}$ & $\mathbf{N P V}_{\mathbf{i j}}$ & DCD $_{\mathrm{ij}}$ & $\mathbf{D C R}_{\mathbf{i j}}$ & Sens $_{i j}$ & Spec $_{i j}$ & $\mathbf{P P V}_{\mathrm{ij}}$ & $\mathrm{NPV}_{\mathrm{ij}}$ & & \\
\hline \multirow[t]{3}{*}{1} & 0.8810 & 0.9900 & 0.8290 & 0.9940 & 0.0001 & 1.0001 & 0.8903 & 0.9909 & 0.8156 & 0.9934 & \multirow{2}{*}{\multicolumn{2}{|c|}{$\begin{array}{l}\text { Manski, C. F. (2020). Bounding the Predictive Values of COVID- } \\
19 \text { Antibodv Tests (No w27226) National Bureau of Economic }\end{array}$}} \\
\hline & 0.9380 & 0.9600 & 0.5520 & 0.9970 & 0.0002 & 1.0003 & 0.9446 & 0.9644 & 0.5221 & 0.9966 & & \\
\hline & 0.8800 & 0.9880 & 0.7940 & 0.9940 & 0.0001 & 1.0001 & 0.8858 & 0.9886 & 0.7847 & 0.9937 & $\begin{array}{l}\text { Research. Available at: } \\
\text { https://www.nber.org/system/files/working_papers/w27226/w2722 } \\
\text { 6.pdf. }\end{array}$ & \\
\hline \multirow[t]{2}{*}{2} & 0.9500 & 0.7800 & 0.5070 & 0.9850 & 0.0000 & 1.0000 & 0.9501 & 0.7804 & 0.5064 & 0.9850 & \multirow{2}{*}{$\begin{array}{l}\text { Ji D, Zhang D, Xu J, Chen Z, Yang T, Zhao P, Chen G, Cheng G, } \\
\text { Wang Y, Bi J, Tan L. Prediction for progression risk in patients } \\
\text { with COVID-19 pneumonia: the CALL score. Clinical Infectious } \\
\text { Diseases. 2020;71(6):1393-1399. }\end{array}$} & Table 4 \\
\hline & 0.4500 & 0.9700 & 0.7830 & 0.1190 & -0.0819 & -1.0931 & 0.0148 & 0.3733 & 0.9949 & 0.8800 & & \\
\hline \multirow[t]{4}{*}{3} & 0.7730 & 1.0000 & 1.0000 & 0.8000 & 0.0000 & 1.0000 & \#DIV/0! & 1.0000 & 1.0000 & \#DIV/0! & \multirow{4}{*}{$\begin{array}{l}\text { Xiang F, Wang X, He X, Peng Z, Yang B, Zhang J, Zhou Q, Ye H, } \\
\text { Ma Y, Li H, Wei X. Antibody detection and dynamic characteristics } \\
\text { in patients with coronavirus disease 2019. Clinical Infectious } \\
\text { Diseases. 2020;71(8):1930-1934. }\end{array}$} & \\
\hline & 0.8330 & 0.9500 & 0.9480 & 0.8380 & 0.0000 & 0.9999 & 0.8323 & 0.9498 & 0.9482 & 0.8387 & & \\
\hline & 0.8750 & 1.0000 & 1.0000 & 0.9520 & 0.0000 & 1.0000 & \#DIV/O! & 1.0000 & 1.0000 & \#DIV/O! & & \\
\hline & 0.7080 & 0.9660 & 0.8500 & 0.8910 & -0.0037 & 0.9928 & 0.6198 & 0.9503 & 0.8939 & 0.9240 & & \\
\hline \multirow[t]{18}{*}{4} & 0.9176 & 1.0000 & 1.0000 & 0.9739 & 0.0000 & 1.0000 & \#DIV/0! & 1.0000 & 1.0000 & \#DIV/0! & \multirow{18}{*}{$\begin{array}{l}\text { Bisoffi Z, Pomari E, Deiana M, Piubelli C, Ronzoni N, Beltrame A, } \\
\text { Bertoli G, Riccardi N, Perandin F, Formenti F, Gobbi F. } \\
\text { Sensitivity, specificity and predictive values of molecular and } \\
\text { serological tests for COVID-19: a longitudinal study in emergency } \\
\text { room. Diagnostics. 2020;10(669):1-12. }\end{array}$} & Fiq. 3 \\
\hline & 0.7619 & 0.9962 & 0.9846 & 0.9286 & 0.0000 & 1.0000 & 0.7603 & 0.9962 & 0.9847 & 0.9292 & & \\
\hline & 0.6118 & 0.9962 & 0.9811 & 0.8874 & 0.0000 & 1.0000 & 0.6095 & 0.9962 & 0.9813 & 0.8884 & & \\
\hline & 0.9412 & 0.9923 & 0.9756 & 0.9811 & 0.0000 & 1.0000 & 0.9415 & 0.9923 & 0.9755 & 0.9810 & & \\
\hline & 0.9412 & 0.9579 & 0.8791 & 0.9804 & 0.0000 & 1.0000 & 0.9411 & 0.9578 & 0.8792 & 0.9804 & & \\
\hline & 0.9167 & 0.9885 & 0.9625 & 0.9736 & 0.0000 & 1.0000 & 0.9168 & 0.9885 & 0.9625 & 0.9736 & & \\
\hline & 0.8941 & 0.9502 & 0.8539 & 0.9650 & 0.0000 & 1.0000 & 0.8941 & 0.9502 & 0.8539 & 0.9650 & & \\
\hline & 0.7857 & 0.9923 & 0.9706 & 0.9350 & 0.0000 & 1.0000 & 0.7866 & 0.9923 & 0.9705 & 0.9347 & & \\
\hline & 0.6235 & 0.9923 & 0.9636 & 0.8900 & 0.0000 & 1.0000 & 0.6243 & 0.9923 & 0.9635 & 0.8896 & & \\
\hline & 0.4588 & 0.7969 & 0.4239 & 0.8189 & 0.0000 & 1.0001 & 0.4589 & 0.7969 & 0.4238 & 0.8189 & & Fig. 5 \\
\hline & 0.4235 & 0.8697 & 0.5143 & 0.8225 & 0.0000 & 1.0002 & 0.4237 & 0.8698 & 0.5141 & 0.8224 & & \\
\hline & 0.3765 & 0.9234 & 0.6154 & 0.8197 & 0.0000 & 0.9999 & 0.3763 & 0.9234 & 0.6156 & 0.8198 & & \\
\hline & 0.3765 & 0.9234 & 0.6154 & 0.8197 & 0.0000 & 0.9999 & 0.3763 & 0.9234 & 0.6156 & 0.8198 & & \\
\hline & 0.3529 & 0.8544 & 0.4412 & 0.8022 & 0.0000 & 1.0003 & 0.3530 & 0.8545 & 0.4411 & 0.8021 & & \\
\hline & 0.3412 & 0.9617 & 0.7436 & 0.8176 & 0.0000 & 1.0000 & 0.3411 & 0.9617 & 0.7437 & 0.8177 & & \\
\hline & 0.3059 & 0.9540 & 0.6842 & 0.8084 & 0.0000 & 1.0000 & 0.3059 & 0.9540 & 0.6842 & 0.8084 & & \\
\hline & 0.2941 & 0.9693 & 0.7576 & 0.8083 & 0.0000 & 1.0001 & 0.2945 & 0.9694 & 0.7573 & 0.8080 & & \\
\hline & 0.2941 & 0.9655 & 0.7353 & 0.8077 & 0.0000 & 1.0001 & 0.2942 & 0.9655 & 0.7352 & 0.8076 & & \\
\hline
\end{tabular}


Rushdi and Serag; IJPR, 6(2): 28-49, 2021; Article no.IJPR. 65260

\begin{tabular}{|c|c|c|c|c|c|c|c|c|c|c|c|c|}
\hline \multirow[t]{17}{*}{ \# } & \multicolumn{4}{|c|}{ Original values } & \multicolumn{2}{|c|}{$\begin{array}{l}\text { Checking } \\
\text { Values }\end{array}$} & \multicolumn{4}{|c|}{ Computed values } & \multirow[t]{2}{*}{ Source } & \multirow{2}{*}{$\begin{array}{l}\text { Data } \\
\text { location } \\
\text { in } \\
\text { Source }\end{array}$} \\
\hline & $\operatorname{Sens}_{\mathrm{ij}}$ & Spec $_{i j}$ & $\mathbf{P P V}_{\mathrm{ij}}$ & $\mathrm{NPV}_{\mathrm{ij}}$ & DCD $_{i j}$ & $\mathrm{DCR}_{\mathrm{ij}}$ & Sens $_{i j}$ & Spec $_{i j}$ & $\mathbf{P P V}_{\mathrm{ij}}$ & $\mathrm{NPV}_{\mathrm{ij}}$ & & \\
\hline & 0.2824 & 0.9464 & 0.6316 & 0.8019 & 0.0000 & 0.9998 & 0.2822 & 0.9463 & 0.6319 & 0.8021 & & \\
\hline & 0.2471 & 0.9847 & 0.8400 & 0.8006 & 0.0000 & 0.9999 & 0.2467 & 0.9847 & 0.8403 & 0.8009 & & \\
\hline & 0.2118 & 0.9732 & 0.7200 & 0.7913 & 0.0000 & 0.9999 & 0.2117 & 0.9732 & 0.7202 & 0.7914 & & \\
\hline & 0.5714 & 0.8857 & 0.6250 & 0.8611 & 0.0000 & 1.0000 & 0.5714 & 0.8857 & 0.6250 & 0.8611 & & Fig. 6 \\
\hline & 0.5429 & 0.8952 & 0.6333 & 0.8545 & 0.0000 & 1.0000 & 0.5428 & 0.8952 & 0.6334 & 0.8545 & & \\
\hline & 0.5429 & 0.8381 & 0.5278 & 0.8462 & 0.0000 & 1.0000 & 0.5430 & 0.8381 & 0.5277 & 0.8462 & & \\
\hline & 0.5143 & 0.9238 & 0.6923 & 0.8509 & 0.0000 & 1.0000 & 0.5144 & 0.9238 & 0.6923 & 0.8509 & & \\
\hline & 0.5143 & 0.7619 & 0.4186 & 0.8247 & 0.0000 & 0.9999 & 0.5142 & 0.7618 & 0.4187 & 0.8247 & & \\
\hline & 0.4857 & 0.9429 & 0.7391 & 0.8462 & 0.0000 & 1.0000 & 0.4856 & 0.9429 & 0.7392 & 0.8463 & & \\
\hline & 0.4857 & 0.8571 & 0.5313 & 0.8333 & 0.0000 & 1.0001 & 0.4858 & 0.8571 & 0.5312 & 0.8332 & & \\
\hline & 0.4571 & 0.9429 & 0.7273 & 0.8390 & 0.0000 & 1.0000 & 0.4570 & 0.9429 & 0.7274 & 0.8390 & & \\
\hline & 0.4571 & 0.9238 & 0.6667 & 0.8362 & 0.0000 & 1.0000 & 0.4572 & 0.9238 & 0.6666 & 0.8361 & & \\
\hline & 0.3714 & 0.9619 & 0.7647 & 0.8211 & 0.0000 & 1.0000 & 0.3714 & 0.9619 & 0.7647 & 0.8211 & & \\
\hline & 0.3714 & 0.9333 & 0.6500 & 0.8167 & 0.0000 & 1.0001 & 0.3716 & 0.9334 & 0.6498 & 0.8166 & & \\
\hline & 0.3143 & 0.9714 & 0.7857 & 0.8095 & 0.0000 & 1.0000 & 0.3145 & 0.9714 & 0.7856 & 0.8094 & & \\
\hline \multirow[t]{6}{*}{5} & 0.8500 & 0.7700 & 0.5480 & 0.9400 & 0.0000 & 1.0001 & 0.8502 & 0.7702 & 0.5477 & 0.9399 & \multirow{6}{*}{$\begin{array}{l}\text { Cui S, Chen S, Li X, Liu S, Wang F. Prevalence of venous } \\
\text { thromboembolism in patients with severe novel coronavirus } \\
\text { pneumonia. Journal of Thrombosis and Haemostasis. } \\
2020 ; 18(6): 1421-1424 \text {. }\end{array}$} & Table 3 \\
\hline & 0.8500 & 0.8800 & 0.7080 & 0.9470 & 0.0005 & 1.0010 & 0.8552 & 0.8843 & 0.6993 & 0.9449 & & \\
\hline & 0.8000 & 0.9020 & 0.7270 & 0.9320 & -0.0001 & 0.9998 & 0.7986 & 0.9012 & 0.7287 & 0.9325 & & \\
\hline & 0.7000 & 0.9340 & 0.7780 & 0.9050 & 0.0002 & 1.0003 & 0.7023 & 0.9347 & 0.7761 & 0.9041 & & \\
\hline & 0.7000 & 0.9670 & 0.8750 & 0.9080 & 0.0001 & 1.0002 & 0.7022 & 0.9673 & 0.8739 & 0.9071 & & \\
\hline & 0.6500 & 0.9670 & 0.8670 & 0.8940 & 0.0001 & 1.0002 & 0.6523 & 0.9673 & 0.8658 & 0.8930 & & \\
\hline \multirow[t]{14}{*}{6} & 1.0000 & 0.2500 & 0.9400 & 1.0000 & 0.0000 & 1.0000 & 1.0000 & \#DIV/0! & \#DIV/O! & 1.0000 & \multirow{14}{*}{$\begin{array}{l}\text { Ni Q, Sun ZY, Qi L, Chen W, Yang Y, Wang L, Zhang X, Yang L, } \\
\text { Fang Y, Xing Z, Zhou Z. A deep learning approach to characterize } \\
2019 \text { coronavirus disease (COVID-19) pneumonia in chest CT } \\
\text { images. European Radiology. 2020;30(12):6517-6527. }\end{array}$} & Table 2 \\
\hline & 0.9400 & 1.0000 & 1.0000 & 0.6200 & 0.0000 & 1.0000 & \#DIV/0! & 1.0000 & 1.0000 & \#DIV/O! & & \\
\hline & 0.9300 & 0.7500 & 0.9800 & 0.5000 & 0.0016 & 1.0048 & 0.9423 & 0.7867 & 0.9755 & 0.4486 & & \\
\hline & 0.8900 & 1.0000 & 1.0000 & 0.4400 & 0.0000 & 1.0000 & \#DIV/0! & 1.0000 & 1.0000 & \#DIV/0! & & \\
\hline & 0.9600 & 0.6300 & 0.7800 & 0.9300 & 0.0014 & 1.0033 & 0.9651 & 0.6625 & 0.7547 & 0.9202 & & \\
\hline & 0.8700 & 0.9000 & 0.9200 & 0.8300 & -0.0007 & 0.9988 & 0.8619 & 0.8935 & 0.9250 & 0.8397 & & \\
\hline & 0.8400 & 0.9300 & 0.9400 & 0.8000 & -0.0010 & 0.9984 & 0.8251 & 0.9227 & 0.9458 & 0.8166 & & \\
\hline & 0.8200 & 0.9600 & 0.9700 & 0.7900 & 0.0006 & 1.0009 & 0.8352 & 0.9639 & 0.9667 & 0.7718 & & \\
\hline & 0.9600 & 0.6600 & 0.7900 & 0.9300 & 0.0007 & 1.0015 & 0.9626 & 0.6756 & 0.7781 & 0.9253 & & Table 3 \\
\hline & 0.9100 & 0.9000 & 0.9300 & 0.8800 & 0.0005 & 1.0007 & 0.9154 & 0.9060 & 0.9254 & 0.8726 & & \\
\hline & 0.9300 & 0.9300 & 0.9400 & 0.9000 & -0.0010 & 0.9986 & 0.9139 & 0.9139 & 0.9515 & 0.9185 & & \\
\hline & 0.9100 & 0.9500 & 0.9600 & 0.8900 & 0.0000 & 1.0001 & 0.9109 & 0.9505 & 0.9596 & 0.8889 & & \\
\hline & 0.9400 & 0.6700 & 0.7600 & 0.9100 & 0.0001 & 1.0002 & 0.9404 & 0.6715 & 0.7588 & 0.9095 & & \\
\hline & 0.8200 & 0.8900 & 0.8900 & 0.8200 & 0.0000 & 1.0000 & 0.8200 & 0.8900 & 0.8900 & 0.8200 & & \\
\hline
\end{tabular}


Rushdi and Serag; IJPR, 6(2): 28-49, 2021; Article no.IJPR. 65260

\begin{tabular}{|c|c|c|c|c|c|c|c|c|c|c|c|c|}
\hline \multirow[t]{28}{*}{ \# } & \multicolumn{4}{|c|}{ Original values } & \multicolumn{2}{|c|}{$\begin{array}{c}\text { Checking } \\
\text { Values }\end{array}$} & \multicolumn{4}{|c|}{ Computed values } & \multirow[t]{2}{*}{ Source } & \multirow{2}{*}{$\begin{array}{l}\text { Data } \\
\text { location } \\
\text { in } \\
\text { Source }\end{array}$} \\
\hline & Sens $_{i j}$ & Spec $_{i j}$ & $\mathbf{P P V}_{\mathrm{ij}}$ & $\mathrm{NPV}_{\mathrm{ij}}$ & DCD $_{i j}$ & $\mathrm{DCR}_{\mathrm{ij}}$ & $\operatorname{Sens}_{\mathrm{ij}}$ & Spec $_{i j}$ & $\mathbf{P P V}_{\mathrm{ij}}$ & $\mathrm{NPV}_{\mathbf{i j}}$ & & \\
\hline & 0.7800 & 0.9600 & 0.9500 & 0.8000 & -0.0008 & 0.9986 & 0.7600 & 0.9554 & 0.9551 & 0.8175 & & \\
\hline & 0.7600 & 0.9300 & 0.9300 & 0.7800 & 0.0013 & 1.0026 & 0.7800 & 0.9370 & 0.9223 & 0.7600 & & \\
\hline & 0.9800 & 0.4600 & 0.7300 & 0.9500 & 0.0014 & 1.0046 & 0.9837 & 0.5118 & 0.6872 & 0.9392 & & \\
\hline & 0.8600 & 0.8700 & 0.9100 & 0.8100 & 0.0006 & 1.0012 & 0.8656 & 0.8753 & 0.9060 & 0.8026 & & \\
\hline & 0.8400 & 0.9000 & 0.9200 & 0.8000 & -0.0003 & 0.9994 & 0.8364 & 0.8976 & 0.9220 & 0.8043 & & \\
\hline & 0.7900 & 0.9700 & 0.9800 & 0.7600 & 0.0010 & 1.0018 & 0.8276 & 0.9763 & 0.9746 & 0.7128 & & \\
\hline & 0.9600 & 0.7000 & 0.8000 & 0.9400 & 0.0010 & 1.0019 & 0.9641 & 0.7231 & 0.7814 & 0.9333 & & \\
\hline & 0.8900 & 0.8800 & 0.9000 & 0.8600 & -0.0007 & 0.9987 & 0.8829 & 0.8723 & 0.9062 & 0.8683 & & \\
\hline & 0.7700 & 0.9100 & 0.9100 & 0.7600 & -0.0008 & 0.9983 & 0.7600 & 0.9053 & 0.9145 & 0.7700 & & \\
\hline & 0.7900 & 0.9800 & 0.9700 & 0.7900 & -0.0017 & 0.9972 & 0.7128 & 0.9700 & 0.9800 & 0.8508 & & \\
\hline & 0.9700 & 0.6100 & 0.8400 & 0.9000 & -0.0006 & 0.9986 & 0.9680 & 0.5937 & 0.8489 & 0.9060 & & \\
\hline & 0.8600 & 0.9400 & 0.9700 & 0.7600 & 0.0004 & 1.0006 & 0.8673 & 0.9434 & 0.9681 & 0.7485 & & \\
\hline & 0.8600 & 0.9400 & 0.9700 & 0.7600 & 0.0004 & 1.0006 & 0.8673 & 0.9434 & 0.9681 & 0.7485 & & \\
\hline & 0.8500 & 0.9700 & 0.9800 & 0.7500 & -0.0008 & 0.9986 & 0.8197 & 0.9629 & 0.9839 & 0.7890 & & \\
\hline & 0.9400 & 1.0000 & 1.0000 & 0.6200 & 0.0000 & 1.0000 & \#DIV/O! & 1.0000 & 1.0000 & \#DIV/0! & & Table 5 \\
\hline & 0.9800 & 1.0000 & 1.0000 & 0.8000 & 0.0000 & 1.0000 & \#DIV/0! & 1.0000 & 1.0000 & \#DIV/O! & & \\
\hline & 0.9300 & 0.7500 & 0.9800 & 0.5000 & 0.0016 & 1.0048 & 0.9423 & 0.7867 & 0.9755 & 0.4486 & & \\
\hline & 0.9700 & 0.8800 & 0.9900 & 0.7000 & -0.0001 & 0.9999 & 0.9692 & 0.8772 & 0.9903 & 0.7055 & & \\
\hline & 0.8900 & 1.0000 & 1.0000 & 0.4400 & 0.0000 & 1.0000 & \#DIV/O! & 1.0000 & 1.0000 & \#DIV/0! & & \\
\hline & 0.9700 & 1.0000 & 1.0000 & 0.7300 & 0.0000 & 1.0000 & \#DIV/O! & 1.0000 & 1.0000 & \#DIV/0! & & \\
\hline & 0.8700 & 0.9000 & 0.9200 & 0.8300 & -0.0007 & 0.9988 & 0.8619 & 0.8935 & 0.9250 & 0.8397 & & \\
\hline & 0.9100 & 0.9300 & 0.9400 & 0.8800 & -0.0009 & 0.9987 & 0.8963 & 0.9191 & 0.9482 & 0.8956 & & \\
\hline & 0.8400 & 0.9300 & 0.9400 & 0.8000 & -0.0010 & 0.9984 & 0.8251 & 0.9227 & 0.9458 & 0.8166 & & \\
\hline & 0.9400 & 0.9400 & 0.9500 & 0.9100 & -0.0009 & 0.9989 & 0.9246 & 0.9246 & 0.9604 & 0.9282 & & \\
\hline & 0.8200 & 0.9600 & 0.9700 & 0.7900 & 0.0006 & 1.0009 & 0.8352 & 0.9639 & 0.9667 & 0.7718 & & \\
\hline & 0.8800 & 0.9500 & 0.9600 & 0.8600 & 0.0003 & 1.0004 & 0.8858 & 0.9526 & 0.9578 & 0.8531 & & \\
\hline \multirow[t]{3}{*}{7} & 0.9300 & 0.5300 & 0.9200 & 0.4200 & -0.0102 & 0.9429 & 0.8807 & 0.3853 & 0.9539 & 0.5657 & \multirow{3}{*}{$\begin{array}{l}\text { Wen Z, Chi Y, Zhang L, Liu H, Du K, Li Z \& Wang D. (2020). } \\
\text { Coronavirus disease 2019: initial detection on chest CT in a } \\
\text { retrospective multicenter study of } 103 \text { Chinese subjects. } \\
\text { Radiology: Cardiothoracic Imaging, 2(2), e200092. }\end{array}$} & Table 3 \\
\hline & 0.9200 & 0.6300 & 0.9300 & 0.5600 & -0.0024 & 0.9915 & 0.9085 & 0.5952 & 0.9390 & 0.5958 & & \\
\hline & 0.9500 & 0.4300 & 0.9000 & 0.6000 & -0.0009 & 0.9954 & 0.9471 & 0.4154 & 0.9053 & 0.6143 & & \\
\hline \multirow[t]{5}{*}{8} & 0.9700 & 0.2500 & 0.6500 & 0.8300 & -0.0023 & 0.9807 & 0.9645 & 0.2190 & 0.6882 & 0.8530 & \multirow{5}{*}{$\begin{array}{l}\text { Ai T, Yang Z, Hou H, Zhan C, Chen C, Lv W \& Xia L. (2020). } \\
\text { Correlation of chest CT and RT-PCR testing in coronavirus } \\
\text { disease } 2019 \text { (COVID-19) in China: a report of } 1014 \text { cases. } \\
\text { Radiology, } 200642 .\end{array}$} & Table 2 \\
\hline & 0.9600 & 0.2700 & 0.6200 & 0.8400 & -0.0006 & 0.9954 & 0.9586 & 0.2630 & 0.6284 & 0.8447 & & \\
\hline & 0.9700 & 0.2200 & 0.7200 & 0.8000 & 0.0015 & 1.0140 & 0.9733 & 0.2413 & 0.6951 & 0.7801 & & \\
\hline & 0.9600 & 0.1900 & 0.6500 & 0.7500 & -0.0002 & 0.9977 & 0.9596 & 0.1884 & 0.6524 & 0.7519 & & \\
\hline & 0.9700 & 0.3000 & 0.6600 & 0.8900 & 0.0015 & 1.0092 & 0.9734 & 0.3269 & 0.6314 & 0.8771 & & \\
\hline
\end{tabular}


Rushdi and Serag; IJPR, 6(2): 28-49, 2021; Article no.IJPR. 65260

\begin{tabular}{|c|c|c|c|c|c|c|c|c|c|c|c|c|}
\hline \multirow[t]{2}{*}{ \# } & \multicolumn{4}{|c|}{ Original values } & \multicolumn{2}{|c|}{$\begin{array}{l}\text { Checking } \\
\text { Values }\end{array}$} & \multicolumn{4}{|c|}{ Computed values } & \multirow[t]{2}{*}{ Source } & \multirow{2}{*}{$\begin{array}{l}\text { Data } \\
\text { location } \\
\text { in } \\
\text { Source }\end{array}$} \\
\hline & Sens $_{i j}$ & Spec $_{i j}$ & $\mathbf{P P V}_{\mathrm{ij}}$ & $\mathbf{N P V}_{\mathrm{ij}}$ & DCD $_{i j}$ & $\mathrm{DCR}_{\mathrm{ij}}$ & Sens $_{i j}$ & Spec $_{i j}$ & $\mathbf{P P V}_{\mathrm{ij}}$ & $\mathrm{NPV}_{\mathbf{i j}}$ & & \\
\hline \multirow[t]{4}{*}{9} & 0.7900 & 0.8400 & 0.8800 & 0.7200 & -0.0010 & 0.9975 & 0.7822 & 0.8337 & 0.8848 & 0.7292 & \multirow{4}{*}{$\begin{array}{l}\text { Guillo E, Gomez IB, Dangeard S, Bennani S, Saab I, Tordjman M } \\
\text { \& Revel MP. (2020). COVID-19 pneumonia: Diagnostic and } \\
\text { prognostic role of CT based on a retrospective analysis of } 214 \\
\text { consecutive patients from Paris, France. European journal of } \\
\text { radiology, 131, 109209. }\end{array}$} & \\
\hline & 0.8100 & 0.9100 & 0.9300 & 0.7600 & -0.0003 & 0.9994 & 0.8062 & 0.9080 & 0.9316 & 0.7644 & & \\
\hline & 0.9000 & 0.7800 & 0.8900 & 0.8000 & 0.0002 & 1.0005 & 0.9013 & 0.7824 & 0.8886 & 0.7977 & & \\
\hline & 0.9300 & 0.8800 & 0.9400 & 0.8600 & -0.0001 & 0.9999 & 0.9292 & 0.8787 & 0.9407 & 0.8615 & & \\
\hline \multirow[t]{3}{*}{10} & 0.9000 & 0.9000 & 0.3210 & 0.9940 & -0.0001 & 0.9996 & 0.8969 & 0.8969 & 0.3284 & 0.9942 & \multirow{3}{*}{$\begin{array}{l}\text { Kumleben N, Bhopal R, Czypionka T, Gruer L, Kock R, Stebbing } \\
\text { J, Stigler FL. Test, test, test for COVID-19 antibodies: the } \\
\text { importance of sensitivity, specificity and predictive powers. Public } \\
\text { Health. 2020;185:88-90. }\end{array}$} & Table 1 \\
\hline & 0.8000 & 0.9900 & 0.8080 & 0.9890 & -0.0001 & 0.9999 & 0.7926 & 0.9895 & 0.8150 & 0.9895 & & Table 2 \\
\hline & 0.9900 & 0.9900 & 0.8380 & 0.9990 & -0.0001 & 0.9999 & 0.9812 & 0.9812 & 0.9075 & 0.9995 & & Table 3 \\
\hline \multirow[t]{18}{*}{11} & 0.7330 & 0.4130 & 0.3330 & 0.7950 & 0.0001 & 1.0025 & 0.7335 & 0.4136 & 0.3325 & 0.7946 & \multirow{18}{*}{$\begin{array}{l}\text { La Torre G, Massetti AP, Antonelli G, Fimiani C, Fantini M, Marte } \\
\text { M \& Villari, P. (2020). Anosmia and ageusia as predictive signs of } \\
\text { COVID-19 in healthcare workers in Italy: a prospective case- } \\
\text { control study. Journal of Clinical Medicine, } 9(9), 2870 .\end{array}$} & \multirow[t]{18}{*}{ Table 3} \\
\hline & 0.6000 & 0.4670 & 0.3100 & 0.7450 & -0.0001 & 0.9960 & 0.5997 & 0.4667 & 0.3103 & 0.7452 & & \\
\hline & 0.1000 & 0.9870 & 0.7500 & 0.7327 & -0.0002 & 0.9965 & 0.0977 & 0.9867 & 0.7548 & 0.7377 & & \\
\hline & 0.0330 & 0.6270 & 0.0340 & 0.6180 & -0.0001 & 1.0079 & 0.0328 & 0.6253 & 0.0342 & 0.6197 & & \\
\hline & 0.3670 & 0.7470 & 0.3666 & 0.7466 & -0.0002 & 0.9946 & 0.3661 & 0.7463 & 0.3675 & 0.7473 & & \\
\hline & 0.4670 & 0.7600 & 0.4375 & 0.7808 & -0.0001 & 0.9992 & 0.4666 & 0.7597 & 0.4379 & 0.7811 & & \\
\hline & 0.4670 & 0.9330 & 0.7370 & 0.8140 & 0.0001 & 1.0005 & 0.4683 & 0.9333 & 0.7360 & 0.8132 & & \\
\hline & 0.3000 & 0.7200 & 0.3000 & 0.7200 & 0.0000 & 1.0000 & 0.3000 & 0.7200 & 0.3000 & 0.7200 & & \\
\hline & 0.1000 & 0.2000 & 0.3333 & 0.7188 & 0.1687 & -0.0062 & 0.8364 & 0.9200 & 0.0108 & 0.0526 & & \\
\hline & 0.0330 & 0.9330 & 0.1670 & 0.7070 & 0.0001 & 0.9664 & 0.0336 & 0.9341 & 0.1645 & 0.7033 & & \\
\hline & 0.1670 & 0.2000 & 0.4545 & 0.7340 & 0.2175 & -0.0298 & 0.9019 & 0.9198 & 0.0178 & 0.0567 & & \\
\hline & 0.1000 & 0.8200 & 0.1875 & 0.3034 & -0.0372 & 9.1730 & 0.0216 & 0.4750 & 0.5375 & 0.6869 & & \\
\hline & 0.4000 & 0.9200 & 0.6670 & 0.7930 & 0.0000 & 1.0001 & 0.4002 & 0.9201 & 0.6668 & 0.7929 & & \\
\hline & 0.3670 & 0.9600 & 0.7860 & 0.7910 & 0.0000 & 0.9999 & 0.3668 & 0.9600 & 0.7862 & 0.7912 & & \\
\hline & 0.3000 & 0.9470 & 0.9620 & 0.7720 & 0.0251 & 1.1368 & 0.8275 & 0.9950 & 0.6934 & 0.2322 & & \\
\hline & 0.0330 & 1.0000 & 1.0000 & 0.7210 & 0.0000 & 1.0000 & \#DIV/0! & 1.0000 & 1.0000 & 0.0000 & & \\
\hline & 0.5670 & 0.9200 & 0.7390 & 0.8410 & -0.0001 & 0.9996 & 0.5656 & 0.9196 & 0.7401 & 0.8417 & & \\
\hline & 0.4000 & 0.9730 & 0.8570 & 0.8020 & 0.0001 & 1.0004 & 0.4025 & 0.9733 & 0.8557 & 0.8004 & & \\
\hline \multirow[t]{8}{*}{12} & 0.9500 & 0.7196 & 0.3878 & 0.9872 & 0.0000 & 1.0000 & 0.9501 & 0.7200 & 0.3873 & 0.9872 & \multirow{8}{*}{$\begin{array}{l}\text { Liu S, Yao N, Qiu Y \& He C. (2020). Predictive performance of } \\
\text { SOFA and qSOFA for in-hospital mortality in severe novel } \\
\text { coronavirus disease. The American Journal of Emergency } \\
\text { Medicine, 38(10), 2074-2080. }\end{array}$} & \multirow[t]{8}{*}{ Table 4} \\
\hline & 0.9000 & 0.8318 & 0.5000 & 0.9780 & 0.0000 & 1.0000 & 0.8999 & 0.8316 & 0.5003 & 0.9780 & & \\
\hline & 0.7000 & 0.8785 & 0.5185 & 0.9400 & 0.0000 & 1.0000 & 0.7000 & 0.8785 & 0.5185 & 0.9400 & & \\
\hline & 0.5500 & 0.9439 & 0.6471 & 0.9182 & 0.0000 & 1.0000 & 0.5502 & 0.9439 & 0.6469 & 0.9181 & & \\
\hline & 0.2500 & 0.9813 & 0.7143 & 0.8750 & 0.0000 & 1.0000 & 0.2501 & 0.9813 & 0.7142 & 0.8749 & & \\
\hline & 0.2000 & 0.9813 & 0.6667 & 0.8678 & 0.0000 & 1.0001 & 0.2001 & 0.9813 & 0.6665 & 0.8677 & & \\
\hline & 0.7000 & 0.8037 & 0.4000 & 0.9348 & 0.0000 & 1.0001 & 0.7001 & 0.8038 & 0.3999 & 0.9348 & & \\
\hline & 1.0000 & 0.4583 & 0.3953 & 1.0000 & 0.0000 & 1.0000 & 1.0000 & \#DIV/0! & \#DIV/0! & 1.0000 & & \\
\hline
\end{tabular}


Rushdi and Serag; IJPR, 6(2): 28-49, 2021; Article no.IJPR.65260

\begin{tabular}{|c|c|c|c|c|c|c|c|c|c|c|c|c|}
\hline \multirow[t]{10}{*}{ \# } & \multicolumn{4}{|c|}{ Original values } & \multicolumn{2}{|c|}{$\begin{array}{l}\text { Checking } \\
\text { Values }\end{array}$} & \multicolumn{4}{|c|}{ Computed values } & \multirow[t]{2}{*}{ Source } & \multirow{2}{*}{$\begin{array}{l}\text { Data } \\
\text { location } \\
\text { in } \\
\text { Source }\end{array}$} \\
\hline & $\operatorname{Sens}_{\mathrm{ij}}$ & Spec $_{i j}$ & $\mathbf{P P V}_{\mathrm{ij}}$ & $\mathbf{N P V}_{\mathrm{ij}}$ & DCD $_{i j}$ & $\mathrm{DCR}_{\mathrm{ij}}$ & Sens $_{i j}$ & Spec $_{i j}$ & $\mathbf{P P V}_{\mathrm{ij}}$ & $\mathrm{NPV}_{\mathbf{i j}}$ & & \\
\hline & 0.9412 & 0.7292 & 0.5517 & 0.9722 & 0.0000 & 1.0000 & 0.9411 & 0.7289 & 0.5521 & 0.9722 & & \\
\hline & 0.8824 & 0.8542 & 0.6818 & 0.9535 & 0.0000 & 1.0000 & 0.8823 & 0.8541 & 0.6819 & 0.9535 & & \\
\hline & 0.7059 & 0.8958 & 0.7059 & 0.8958 & 0.0000 & 1.0000 & 0.7059 & 0.8958 & 0.7059 & 0.8958 & & \\
\hline & 0.5882 & 0.9585 & 0.8333 & 0.8679 & -0.0001 & 0.9999 & 0.5871 & 0.9583 & 0.8339 & 0.8684 & & \\
\hline & 0.2353 & 0.9792 & 0.8000 & 0.7833 & 0.0000 & 0.9999 & 0.2350 & 0.9792 & 0.8003 & 0.7836 & & \\
\hline & 0.1765 & 0.9792 & 0.7500 & 0.7705 & 0.0000 & 0.9998 & 0.1762 & 0.9792 & 0.7503 & 0.7708 & & \\
\hline & 0.1765 & 1.0000 & 1.0000 & 0.7742 & 0.0000 & 1.0000 & \#DIV/0! & 1.0000 & 1.0000 & \#DIV/0! & & \\
\hline & 0.7059 & 0.8542 & 0.6316 & 0.8913 & 0.0000 & 1.0000 & 0.7058 & 0.8542 & 0.6317 & 0.8913 & & \\
\hline \multirow[t]{14}{*}{13} & 0.6600 & 0.8980 & 0.5320 & 0.9370 & -0.0002 & 0.9993 & 0.6576 & 0.8970 & 0.5347 & 0.9376 & \multirow{14}{*}{$\begin{array}{l}\text { Covino M, Sandroni C, Santoro M, Sabia L, Simeoni B, Bocci MG } \\
\& \text { Franceschi, F. (2020). Predicting intensive care unit admission } \\
\text { and death for COVID-19 patients in the emergency department } \\
\text { using early warning scores. Resuscitation, } 156,84-91 .\end{array}$} & Table 2 \\
\hline & 0.7000 & 0.6480 & 0.2590 & 0.9250 & 0.0001 & 1.0011 & 0.7008 & 0.6488 & 0.2583 & 0.9248 & & \\
\hline & 0.6600 & 0.8490 & 0.4340 & 0.9340 & -0.0001 & 0.9994 & 0.6587 & 0.8483 & 0.4354 & 0.9344 & & \\
\hline & 0.7000 & 0.7850 & 0.3650 & 0.9370 & 0.0001 & 1.0005 & 0.7007 & 0.7856 & 0.3642 & 0.9368 & & \\
\hline & 0.6200 & 0.8270 & 0.4780 & 0.8980 & 0.0009 & 1.0048 & 0.6278 & 0.8317 & 0.4698 & 0.8949 & & \\
\hline & 0.3400 & 0.7960 & 0.2270 & 0.8730 & 0.0001 & 1.0042 & 0.3410 & 0.7967 & 0.2263 & 0.8725 & & \\
\hline & 0.6000 & 0.7500 & 0.2970 & 0.9140 & -0.0001 & 0.9994 & 0.5995 & 0.7496 & 0.2975 & 0.9142 & & \\
\hline & 0.5890 & 0.8960 & 0.5320 & 0.9150 & -0.0002 & 0.9992 & 0.5868 & 0.8952 & 0.5342 & 0.9157 & & \\
\hline & 0.6610 & 0.6480 & 0.2740 & 0.9050 & 0.0000 & 1.0006 & 0.6614 & 0.6484 & 0.2737 & 0.9049 & & \\
\hline & 0.7140 & 0.7730 & 0.3880 & 0.9310 & 0.0001 & 1.0008 & 0.7153 & 0.7741 & 0.3865 & 0.9306 & & \\
\hline & 0.6780 & 0.7910 & 0.3960 & 0.9240 & 0.0000 & 1.0000 & 0.6781 & 0.7910 & 0.3959 & 0.9240 & & \\
\hline & 0.6010 & 0.8420 & 0.5430 & 0.8750 & 0.0010 & 1.0050 & 0.6095 & 0.8467 & 0.5342 & 0.8711 & & \\
\hline & 0.3210 & 0.7950 & 0.2400 & 0.8530 & 0.0000 & 0.9994 & 0.3209 & 0.7949 & 0.2401 & 0.8531 & & \\
\hline & 0.5890 & 0.7550 & 0.3270 & 0.9010 & 0.0000 & 1.0004 & 0.5893 & 0.7552 & 0.3267 & 0.9009 & & \\
\hline \multirow[t]{7}{*}{14} & 0.7895 & 0.7222 & 0.3750 & 0.9420 & 0.0000 & 0.9999 & 0.7894 & 0.7221 & 0.3751 & 0.9420 & \multirow{7}{*}{$\begin{array}{l}\text { Hui TC, Khoo HW, Young BE, Mohideen SMH, Lee YS, Lim J \& } \\
\text { Tan CH. (2020). Clinical utility of chest radiography for severe } \\
\text { COVID-19. Quantitative imaging in medicine and surgery, 10(7), } \\
\text { 1540. }\end{array}$} & Table 4 \\
\hline & 0.8182 & 0.6837 & 0.2250 & 0.9710 & 0.0000 & 0.9999 & 0.8181 & 0.6835 & 0.2251 & 0.9710 & & \\
\hline & 0.6316 & 0.9111 & 0.6000 & 0.9213 & 0.0000 & 1.0000 & 0.6315 & 0.9110 & 0.6001 & 0.9213 & & \\
\hline & 0.7273 & 0.8776 & 0.4000 & 0.9663 & 0.0000 & 1.0000 & 0.7272 & 0.8776 & 0.4001 & 0.9663 & & \\
\hline & 0.9444 & 0.6364 & 0.5152 & 0.9655 & 0.0000 & 1.0000 & 0.9444 & 0.6365 & 0.5151 & 0.9655 & & Table 5 \\
\hline & 0.8889 & 0.9545 & 0.8889 & 0.9545 & 0.0000 & 1.0000 & 0.8889 & 0.9545 & 0.8889 & 0.9545 & & \\
\hline & 1.0000 & 0.8627 & 0.6111 & 1.0000 & 0.0000 & 1.0000 & 1.0000 & \#DIV/O! & \#DIV/O! & 1.0000 & & \\
\hline \multirow[t]{2}{*}{15} & 0.9390 & 0.9600 & 0.9480 & 0.9520 & 0.0000 & 0.9999 & 0.9378 & 0.9592 & 0.9491 & 0.9530 & \multirow{2}{*}{$\begin{array}{l}\text { Gezer NS, Ergan B, Barış MM, Appak Ö, Sayıner AA, Balcı P \& } \\
\text { Kılınç, O. (2020). COVID-19 S: A new proposal for diagnosis and } \\
\text { structured reporting of COVID-19 on computed tomography } \\
\text { imaging. Diagnostic and Interventional Radiology, } 26(4), 315 .\end{array}$} & \\
\hline & 0.9900 & 0.8710 & 0.8580 & 0.9910 & 0.0000 & 1.0000 & 0.9900 & 0.8705 & 0.8586 & 0.9910 & & \\
\hline \multirow[t]{2}{*}{16} & 0.7200 & 0.9400 & 0.9200 & 0.7600 & -0.0012 & 0.9973 & 0.6992 & 0.9340 & 0.9271 & 0.7779 & \multirow{2}{*}{$\begin{array}{l}\text { Bai, H.X, Hsieh B, Xiong Z, Halsey K, Choi JW, Tran TML \& } \\
\text { Jiang, X. L. (2020). Performance of radiologists in differentiating }\end{array}$} & Table 3 \\
\hline & 0.7200 & 0.8800 & 0.8700 & 0.7400 & 0.0002 & 1.0006 & 0.7220 & 0.8811 & 0.8689 & 0.7381 & & \\
\hline
\end{tabular}


Rushdi and Serag; IJPR, 6(2): 28-49, 2021; Article no.IJPR. 65260

\begin{tabular}{|c|c|c|c|c|c|c|c|c|c|c|c|c|}
\hline \multirow[t]{10}{*}{ \# } & \multicolumn{4}{|c|}{ Original values } & \multicolumn{2}{|c|}{$\begin{array}{c}\text { Checking } \\
\text { Values }\end{array}$} & \multicolumn{4}{|c|}{ Computed values } & \multirow[t]{2}{*}{ Source } & \multirow{2}{*}{$\begin{array}{l}\text { Data } \\
\text { location } \\
\text { in } \\
\text { Source }\end{array}$} \\
\hline & Sens $_{i j}$ & Spec $_{\mathrm{ij}}$ & $\mathbf{P P V}_{\mathrm{ij}}$ & $\mathbf{N P V}_{\mathrm{ij}}$ & $\mathbf{D C D}_{\mathrm{ij}}$ & $\mathbf{D C R}_{\mathrm{ij}}$ & Sens $_{i j}$ & $\mathrm{Spec}_{\mathrm{ij}}$ & $\mathbf{P P V}_{\mathrm{ij}}$ & $\mathbf{N P V}_{\mathbf{i j}}$ & & \\
\hline & 0.9400 & 0.2400 & 0.5700 & 0.7900 & 0.0002 & 1.0020 & 0.9404 & 0.2414 & 0.5681 & 0.7887 & \multirow[t]{8}{*}{ COVID-19 from viral pneumonia on chest CT. Radiology, 200823.} & \\
\hline & 0.8000 & 1.0000 & 1.0000 & 0.8200 & 0.0000 & 1.0000 & \#DIV/0! & 1.0000 & 1.0000 & \#DIV/O! & & Table 4 \\
\hline & 0.6700 & 0.9300 & 0.9100 & 0.7200 & -0.0006 & 0.9986 & 0.6618 & 0.9276 & 0.9130 & 0.7274 & & \\
\hline & 0.9700 & 0.0700 & 0.5300 & 0.6700 & -0.0006 & 0.9561 & 0.9682 & 0.0661 & 0.5452 & 0.6834 & & \\
\hline & 0.9300 & 1.0000 & 1.0000 & 0.9300 & 0.0000 & 1.0000 & \#DIV/0! & 1.0000 & 1.0000 & \#DIV/0! & & \\
\hline & 0.8300 & 0.9300 & 0.9300 & 0.8400 & 0.0007 & 1.0011 & 0.8400 & 0.9346 & 0.9251 & 0.8300 & & \\
\hline & 0.7300 & 0.9300 & 0.9200 & 0.7600 & 0.0002 & 1.0004 & 0.7327 & 0.9309 & 0.9190 & 0.7575 & & \\
\hline & 0.7000 & 1.0000 & 1.0000 & 0.7600 & 0.0000 & 1.0000 & \#DIV/0! & 1.0000 & 1.0000 & \#DIV/0! & & \\
\hline \multirow[t]{7}{*}{17} & 0.8300 & 0.3800 & 0.6300 & 0.8900 & 0.0463 & 1.3929 & 0.9574 & 0.7383 & 0.2700 & 0.6373 & \multirow{7}{*}{$\begin{array}{l}\text { Tan C, Huang Y, Shi F, Tan K, Ma Q, Chen Y, Jiang X, Li X. } \\
\text { C-reactive protein correlates with computed tomographic findings } \\
\text { and predicts severe COVID-19 early. Journal of Medical Virology. } \\
2020 ; 92(7): 856-62 \text {. }\end{array}$} & Table 2 \\
\hline & 0.8300 & 0.4300 & 0.2900 & 0.9000 & 0.0000 & 0.9993 & 0.8297 & 0.4295 & 0.2904 & 0.9002 & & \\
\hline & 0.3300 & 0.6700 & 0.2200 & 0.7800 & 0.0000 & \#DIV/0! & 0.3300 & 0.6700 & 0.2200 & 0.7800 & & \\
\hline & 0.8300 & 0.4300 & 0.2900 & 0.9000 & 0.0000 & 0.9993 & 0.8297 & 0.4295 & 0.2904 & 0.9002 & & \\
\hline & 0.8300 & 0.9100 & 0.7100 & 0.9500 & -0.0006 & 0.9987 & 0.8214 & 0.9050 & 0.7221 & 0.9527 & & \\
\hline & 0.8300 & 0.8100 & 0.5600 & 0.9400 & -0.0007 & 0.9978 & 0.8239 & 0.8033 & 0.5706 & 0.9424 & & \\
\hline & 0.5000 & 0.9100 & 0.6000 & 0.8600 & -0.0023 & 0.9893 & 0.4768 & 0.9021 & 0.6221 & 0.8708 & & \\
\hline \multirow[t]{2}{*}{18} & 0.6000 & 0.5400 & 0.6000 & 0.5400 & 0.0000 & 1.0000 & 0.6000 & 0.5400 & 0.6000 & 0.5400 & \multirow{2}{*}{$\begin{array}{l}\text { Caruso D, Zerunian M, Polici M, Pucciarelli F, Polidori T, Rucci C } \\
\& \text { Laghi, A. (2020). Chest CT features of COVID-19 in Rome, } \\
\text { Italy. Radiology, 201237. }\end{array}$} & Table 2 \\
\hline & 0.8899 & 0.4566 & 0.5364 & 0.8799 & 0.0056 & 1.0343 & 0.9098 & 0.5119 & 0.4811 & 0.8544 & & \\
\hline \multirow[t]{2}{*}{19} & 0.8630 & 0.5930 & 0.3390 & 0.9474 & 0.0001 & 1.0008 & 0.8638 & 0.5946 & 0.3376 & 0.9471 & \multirow{2}{*}{$\begin{array}{l}\mathrm{Bi} \text { X, SU Z, Yan H, Du J, Wang J, Chen L \& Li J. (2020). } \\
\text { Prediction of severe illness due to COVID-19 based on an } \\
\text { analysis of initial Fibrinogen to Albumin Ratio and Platelet count. } \\
\text { Platelets, 1-6. }\end{array}$} & Table \\
\hline & 0.8570 & 0.4290 & 0.9000 & 0.3330 & -0.0001 & 0.9994 & 0.8567 & 0.4285 & 0.9002 & 0.3335 & & 3.3 \\
\hline \multirow[t]{2}{*}{20} & 0.8670 & 0.9360 & 0.9110 & 0.9030 & 0.0000 & 1.0000 & 0.8669 & 0.9360 & 0.9110 & 0.9030 & \multirow{2}{*}{$\begin{array}{l}\text { Dangis A, Gieraerts C, Bruecker YD, Janssen L, Valgaeren H, } \\
\text { Obbels D \& Symons R. (2020). Accuracy and reproducibility of } \\
\text { low-dose submillisievert chest CT for the diagnosis of COVID-19. } \\
\text { Radiology: Cardiothoracic Imaging, 2(2), e200196. }\end{array}$} & Table 2 \\
\hline & 0.9560 & 0.9320 & 0.9150 & 0.9650 & 0.0000 & 1.0000 & 0.9559 & 0.9318 & 0.9153 & 0.9651 & & \\
\hline \multirow[t]{9}{*}{21} & 0.5200 & 0.8900 & 0.2600 & 0.9600 & -0.0005 & 0.9949 & 0.5103 & 0.8862 & 0.2675 & 0.9615 & \multirow{9}{*}{$\begin{array}{l}\text { Laguna-Goya R, Utrero-Rico A, Talayero P, Lasa-Lazaro M, } \\
\text { Ramirez-Fernandez A, Naranjo L \& Fernández-Ruiz M. (2020). } \\
\text { 6-based mortality risk model for hospitalized patients with } \\
\text { COVID-19. Journal of Allergy and Clinical Immunology, 146(4), } \\
799-807 .\end{array}$} & \\
\hline & 0.9700 & 0.5300 & 0.1400 & 0.9900 & -0.0025 & 0.9644 & 0.9346 & 0.3326 & 0.2692 & 0.9956 & & \\
\hline & 0.7400 & 0.7800 & 0.1700 & 0.9700 & -0.0049 & 0.9424 & 0.6513 & 0.6994 & 0.2379 & 0.9801 & & \\
\hline & 0.8000 & 0.5300 & 0.1600 & 0.9600 & 0.0002 & 1.0038 & 0.8021 & 0.5333 & 0.1582 & 0.9595 & & \\
\hline & 0.7200 & 0.7100 & 0.1600 & 0.9700 & -0.0003 & 0.9958 & 0.7155 & 0.7055 & 0.1630 & 0.9706 & & \\
\hline & 0.7000 & 0.7500 & 0.1400 & 0.9700 & -0.0034 & 0.9450 & 0.6370 & 0.6929 & 0.1780 & 0.9773 & & \\
\hline & 0.6200 & 0.8400 & 0.2300 & 0.9600 & -0.0026 & 0.9742 & 0.5773 & 0.8146 & 0.2630 & 0.9663 & & \\
\hline & 0.6300 & 0.6100 & 0.1100 & 0.9500 & -0.0020 & 0.9194 & 0.6002 & 0.5797 & 0.1229 & 0.9556 & & \\
\hline & 0.7700 & 0.6700 & 0.1100 & 0.9600 & -0.0104 & 0.7772 & 0.5937 & 0.4698 & 0.2207 & 0.9821 & & \\
\hline
\end{tabular}


Rushdi and Serag; IJPR, 6(2): 28-49, 2021; Article no.IJPR. 65260

\begin{tabular}{|c|c|c|c|c|c|c|c|c|c|c|c|c|}
\hline \multirow[t]{11}{*}{ \# } & \multicolumn{4}{|c|}{ Original values } & \multicolumn{2}{|c|}{$\begin{array}{c}\text { Checking } \\
\text { Values }\end{array}$} & \multicolumn{4}{|c|}{ Computed values } & \multirow[t]{2}{*}{ Source } & \multirow{2}{*}{$\begin{array}{l}\text { Data } \\
\text { location } \\
\text { in } \\
\text { Source }\end{array}$} \\
\hline & Sens $_{i j}$ & Spec $_{i j}$ & $\mathbf{P P V}_{\mathrm{ij}}$ & $\mathbf{N P V}_{\mathbf{i j}}$ & DCD $_{\mathrm{ij}}$ & $\mathbf{D C R}_{\mathrm{ij}}$ & Sens $_{i j}$ & Spec $_{i j}$ & $\mathbf{P P V}_{\mathrm{ij}}$ & $\mathbf{N P V}_{\mathbf{i j}}$ & & \\
\hline & 0.8600 & 0.6200 & 0.1500 & 0.9800 & -0.0012 & 0.9824 & 0.8413 & 0.5847 & 0.1698 & 0.9827 & & \\
\hline & 0.6300 & 0.8300 & 0.1300 & 0.9800 & -0.0011 & 0.9815 & 0.5999 & 0.8113 & 0.1450 & 0.9823 & & \\
\hline & 0.8600 & 0.6800 & 0.1700 & 0.9800 & -0.0022 & 0.9751 & 0.8253 & 0.6203 & 0.2104 & 0.9846 & & \\
\hline & 0.8800 & 0.7500 & 0.3200 & 0.9800 & 0.0004 & 1.0022 & 0.8849 & 0.7587 & 0.3099 & 0.9791 & & \\
\hline & 0.7500 & 0.8800 & 0.3200 & 0.9800 & 0.0004 & 1.0022 & 0.7587 & 0.8849 & 0.3099 & 0.9791 & & \\
\hline & 0.8600 & 0.8300 & 0.2700 & 0.9900 & 0.0012 & 1.0062 & 0.8823 & 0.8563 & 0.2325 & 0.9878 & & \\
\hline & 0.8100 & 0.8800 & 0.3400 & 0.9800 & -0.0018 & 0.9921 & 0.7749 & 0.8555 & 0.3895 & 0.9838 & & \\
\hline & 0.8100 & 0.9200 & 0.4400 & 0.9800 & -0.0018 & 0.9943 & 0.7700 & 0.9003 & 0.5001 & 0.9842 & & \\
\hline & 0.8800 & 0.8900 & 0.3800 & 0.9900 & 0.0001 & 1.0004 & 0.8823 & 0.8922 & 0.3747 & 0.9898 & & \\
\hline \multirow[t]{5}{*}{22} & 0.7690 & 0.7260 & 0.4470 & 0.9160 & 0.0000 & 0.9999 & 0.7689 & 0.7259 & 0.4472 & 0.9161 & \multirow{5}{*}{$\begin{array}{l}\text { Jehi L, Ji X, Milinovich A, Erzurum S, Merlino A, Gordon S \& } \\
\text { Kattan, MW. (2020). Development and validation of a model for } \\
\text { individualized prediction of hospitalization risk in } 4,536 \text { patients } \\
\text { with COVID-19. PloS one, 15(8), e0237419. }\end{array}$} & Table 2 \\
\hline & 0.5190 & 0.9180 & 0.6460 & 0.8960 & 0.0053 & 1.0209 & 0.5841 & 0.9358 & 0.5837 & 0.8688 & & \\
\hline & 0.3880 & 0.9630 & 0.7490 & 0.8460 & -0.0001 & 0.9996 & 0.3864 & 0.9628 & 0.7502 & 0.8469 & & \\
\hline & 0.2530 & 0.9790 & 0.7720 & 0.8200 & -0.0002 & 0.9984 & 0.2486 & 0.9785 & 0.7761 & 0.8234 & & \\
\hline & 0.1170 & 0.9920 & 0.8000 & 0.7960 & -0.0002 & 0.9966 & 0.1118 & 0.9916 & 0.8081 & 0.8042 & & \\
\hline \multirow[t]{4}{*}{23} & 0.5000 & 0.8280 & 0.3130 & 0.9140 & 0.0001 & 1.0015 & 0.5015 & 0.8288 & 0.3117 & 0.9135 & \multirow{4}{*}{$\begin{array}{l}\text { Gruskay JA, Dvorzhinskiy A, Konnaris M.A, LeBrun DG, } \\
\text { Ghahramani GC, Premkumar A \& Ricci WM. (2020). Universal } \\
\text { testing for COVID-19 in essential orthopaedic surgery reveals a } \\
\text { high percentage of asymptomatic infections. JBJS, 102(16), 1379- } \\
1388 .\end{array}$} & Table 2 \\
\hline & 0.5000 & 0.9220 & 0.5000 & 0.9220 & 0.0000 & 1.0000 & 0.5000 & 0.9220 & 0.5000 & 0.9220 & & \\
\hline & 0.4000 & 0.9840 & 0.8000 & 0.9130 & 0.0002 & 1.0006 & 0.4057 & 0.9844 & 0.7962 & 0.9111 & & \\
\hline & 0.6000 & 0.7660 & 0.2860 & 0.9250 & 0.0002 & 1.0016 & 0.6015 & 0.7671 & 0.2848 & 0.9246 & & \\
\hline \multirow[t]{2}{*}{24} & 0.8900 & 0.6600 & 0.6300 & 0.9000 & -0.0005 & 0.9983 & 0.8876 & 0.6545 & 0.6357 & 0.9022 & \multirow{2}{*}{$\begin{array}{l}\text { Gidari A, De Socio G V, Sabbatini S \& Francisci D. (2020). } \\
\text { Predictive value of National Early Warning Score } 2 \text { (NEWS2) for } \\
\text { intensive care unit admission in patients with SARS-CoV-2 } \\
\text { infection. Infectious Diseases, 52(10), 698-704. }\end{array}$} & Table 2 \\
\hline & 0.6300 & 0.9800 & 0.9400 & 0.8000 & -0.0018 & 0.9960 & 0.5612 & 0.9735 & 0.9543 & 0.8419 & & \\
\hline \multirow[t]{6}{*}{25} & 0.6700 & 0.9300 & 0.8000 & 0.8800 & 0.0013 & 1.0031 & 0.6883 & 0.9353 & 0.7862 & 0.8709 & \multirow{6}{*}{$\begin{array}{l}\text { Himoto Y, Sakata A, Kirita M, Hiroi T, Kobayashi KI, Kubo K, Kim } \\
\text { H, Nishimoto A, Maeda C, Kawamura A, Komiya N. Diagnostic } \\
\text { performance of chest CT to differentiate COVID-19 pneumonia in } \\
\text { non-high-epidemic area in Japan. Japanese Journal of Radiology. } \\
2020 ; 38(5): 400-406 .\end{array}$} & Table 3 \\
\hline & 0.8300 & 0.6000 & 0.4500 & 0.9000 & 0.0002 & 1.0009 & 0.8308 & 0.6013 & 0.4486 & 0.8995 & & \\
\hline & 0.8300 & 0.6000 & 0.4500 & 0.9000 & 0.0002 & 1.0009 & 0.8308 & 0.6013 & 0.4486 & 0.8995 & & \\
\hline & 0.8300 & 0.6700 & 0.5000 & 0.9100 & 0.0005 & 1.0022 & 0.8328 & 0.6744 & 0.4950 & 0.9084 & & \\
\hline & 0.8300 & 0.6000 & 0.4500 & 0.9000 & 0.0002 & 1.0009 & 0.8308 & 0.6013 & 0.4486 & 0.8995 & & \\
\hline & 0.8300 & 0.8000 & 0.6300 & 0.9200 & 0.0001 & 1.0001 & 0.8304 & 0.8004 & 0.6294 & 0.9198 & & \\
\hline \multirow[t]{6}{*}{26} & 0.4118 & 0.9215 & 0.2800 & 0.9548 & 0.0000 & 0.9999 & 0.4117 & 0.9215 & 0.2801 & 0.9548 & \multirow{6}{*}{$\begin{array}{l}\text { Luo Y, Mao L, Yuan X, Xue Y, Lin Q, Tang G \& Sun Z. (2020) } \\
\text { Prediction model based on the combination of cytokines and } \\
\text { lymphocyte subsets for prognosis of SARS-CoV-2 infection. } \\
\text { Journal of Clinical Immunology, 40(7), 960-969. }\end{array}$} & Table 2 \\
\hline & 0.6863 & 0.9041 & 0.3465 & 0.9749 & 0.0000 & 0.9999 & 0.6860 & 0.9040 & 0.3468 & 0.9749 & & \\
\hline & 0.5490 & 0.9026 & 0.2947 & 0.9643 & 0.0000 & 1.0000 & 0.5491 & 0.9026 & 0.2946 & 0.9643 & & \\
\hline & 0.3333 & 0.9012 & 0.2000 & 0.9480 & 0.0000 & 0.9999 & 0.3332 & 0.9012 & 0.2001 & 0.9480 & & \\
\hline & 0.3922 & 0.9070 & 0.2381 & 0.9527 & 0.0000 & 1.0000 & 0.3922 & 0.9070 & 0.2381 & 0.9527 & & \\
\hline & 0.7843 & 0.9041 & 0.3774 & 0.9826 & 0.0000 & 1.0000 & 0.7841 & 0.9040 & 0.3777 & 0.9826 & & \\
\hline
\end{tabular}


Rushdi and Serag; IJPR, 6(2): 28-49, 2021; Article no.IJPR. 65260

\begin{tabular}{|c|c|c|c|c|c|c|c|c|c|c|c|c|}
\hline \multirow[t]{5}{*}{ \# } & \multicolumn{4}{|c|}{ Original values } & \multicolumn{2}{|c|}{$\begin{array}{l}\text { Checking } \\
\text { Values }\end{array}$} & \multicolumn{4}{|c|}{ Computed values } & \multirow[t]{2}{*}{ Source } & \multirow{2}{*}{$\begin{array}{l}\text { Data } \\
\text { location } \\
\text { in } \\
\text { Source }\end{array}$} \\
\hline & $\operatorname{Sen}_{\mathrm{ij}}$ & Spec $_{i j}$ & $\mathbf{P P V}_{\mathrm{ij}}$ & $\mathrm{NPV}_{\mathrm{ij}}$ & $\mathrm{DCD}_{\mathrm{ij}}$ & $\mathbf{D C R}_{\mathrm{ij}}$ & Sens $_{\mathrm{ij}}$ & Spec $_{i j}$ & $\mathbf{P P V}_{\mathrm{ij}}$ & $\mathrm{NPV}_{\mathrm{ij}}$ & & \\
\hline & 0.7255 & 0.9099 & 0.3737 & 0.9781 & 0.0000 & 0.9999 & 0.7252 & 0.9098 & 0.3741 & 0.9781 & & \\
\hline & 0.6667 & 0.9055 & 0.3434 & 0.9734 & 0.0000 & 0.9999 & 0.6664 & 0.9054 & 0.3437 & 0.9734 & & \\
\hline & 0.9020 & 0.9026 & 0.4071 & 0.9920 & 0.0000 & 1.0000 & 0.9018 & 0.9024 & 0.4075 & 0.9920 & & \\
\hline \multirow[t]{11}{*}{27} & 0.8934 & 0.9016 & 0.9008 & 0.8943 & 0.0000 & 1.0000 & 0.8934 & 0.9016 & 0.9008 & 0.8943 & \multirow{11}{*}{$\begin{array}{l}\text { Ardakani AA, Acharya UR, Habibollahi \& Mohammadi, A. (2021). } \\
\text { COVIDiag: A clinical CAD system to diagnose COVID-19 } \\
\text { pneumonia based on CT findings. European radiology, 31(1), } \\
\text { 121-130. }\end{array}$} & \multirow[t]{11}{*}{ Table 2} \\
\hline & 0.7903 & 0.8710 & 0.8596 & 0.8060 & 0.0000 & 1.0000 & 0.7902 & 0.8710 & 0.8596 & 0.8061 & & \\
\hline & 0.9098 & 0.8975 & 0.8987 & 0.9087 & 0.0000 & 1.0000 & 0.9098 & 0.8975 & 0.8987 & 0.9087 & & \\
\hline & 0.8871 & 0.8064 & 0.8209 & 0.8772 & 0.0000 & 1.0000 & 0.8871 & 0.8065 & 0.8208 & 0.8772 & & \\
\hline & 0.8852 & 0.9344 & 0.9310 & 0.8906 & 0.0000 & 1.0000 & 0.8852 & 0.9344 & 0.9310 & 0.8906 & & \\
\hline & 0.8548 & 0.8871 & 0.8833 & 0.8594 & 0.0000 & 1.0000 & 0.8548 & 0.8871 & 0.8833 & 0.8594 & & \\
\hline & 0.9344 & 0.9221 & 0.9231 & 0.9336 & 0.0000 & 1.0000 & 0.9345 & 0.9222 & 0.9230 & 0.9335 & & \\
\hline & 0.8871 & 0.8710 & 0.8730 & 0.8852 & 0.0000 & 1.0000 & 0.8870 & 0.8709 & 0.8731 & 0.8853 & & \\
\hline & 0.9467 & 0.9303 & 0.9316 & 0.9458 & 0.0000 & 1.0000 & 0.9468 & 0.9305 & 0.9314 & 0.9457 & & \\
\hline & 0.9354 & 0.9032 & 0.9063 & 0.9333 & 0.0000 & 1.0000 & 0.9355 & 0.9034 & 0.9062 & 0.9332 & & \\
\hline & 0.8710 & 0.8871 & 0.8824 & 0.8730 & -0.0003 & 0.9995 & 0.8678 & 0.8842 & 0.8853 & 0.8761 & & \\
\hline \multirow[t]{4}{*}{28} & 0.8700 & 0.8000 & 0.3400 & 0.9800 & -0.0005 & 0.9977 & 0.8632 & 0.7904 & 0.3533 & 0.9811 & \multirow{4}{*}{$\begin{array}{l}\text { Larner AJ. (2021). Cognitive testing in the COVID-19 era: can } \\
\text { existing screeners be adapted for telephone use?. } \\
\text { Neurodegenerative Disease Management, } 11(1), 77-82 .\end{array}$} & \multirow[t]{4}{*}{ Table 3} \\
\hline & 0.8000 & 0.8900 & 0.4600 & 0.9700 & -0.0017 & 0.9944 & 0.7729 & 0.8732 & 0.5002 & 0.9744 & & \\
\hline & 0.8400 & 0.5200 & 0.4900 & 0.8600 & 0.0012 & 1.0078 & 0.8449 & 0.5292 & 0.4808 & 0.8555 & & \\
\hline & 0.5600 & 0.8400 & 0.6600 & 0.7700 & -0.0010 & 0.9950 & 0.5531 & 0.8362 & 0.6662 & 0.7749 & & \\
\hline \multirow[t]{4}{*}{29} & 0.9400 & 0.7300 & 0.8100 & 0.9000 & -0.0012 & 0.9975 & 0.9342 & 0.7101 & 0.8248 & 0.9086 & \multirow{4}{*}{$\begin{array}{l}\text { Rueckel J, Fink N, Kaestle S, Stüber T, Schwarze V, Gresser E, } \\
\text { Hoppe BF, Rudolph J, Kunz WG, Ricke J, Sabel BO. COVID-19 } \\
\text { Pandemic and Upcoming Influenza Season-Does an Expert's } \\
\text { Computed Tomography Assessment Differentially Identify COVID- } \\
\text { 19, Influenza and Pneumonias of Other Origin?. Journal of } \\
\text { Clinical Medicine. 2021;10(84):1-13. }\end{array}$} & \multirow[t]{4}{*}{ Table 2} \\
\hline & 0.7100 & 0.9800 & 0.9800 & 0.7300 & 0.0004 & 1.0008 & 0.7300 & 0.9819 & 0.9780 & 0.7100 & & \\
\hline & 0.9300 & 0.7100 & 0.8000 & 0.9000 & 0.0014 & 1.0031 & 0.9363 & 0.7304 & 0.7833 & 0.8905 & & \\
\hline & 0.7400 & 0.9800 & 0.9800 & 0.7500 & 0.0002 & 1.0004 & 0.7500 & 0.9810 & 0.9789 & 0.7400 & & \\
\hline \multirow[t]{4}{*}{30} & 0.6300 & 0.9250 & 0.9440 & 0.5520 & -0.0002 & 0.9994 & 0.6274 & 0.9242 & 0.9446 & 0.5547 & \multirow{4}{*}{$\begin{array}{l}\text { Liu J, Lian R, Zhang G, Hou B, Wang C, Dong J \& Ye, T. (2021). } \\
\text { Changes in serum virus-specific IgM/lgG antibody in } \\
\text { asymptomatic and discharged patients with reoccurring positive } \\
\text { COVID-19 nucleic acid test (RPNAT). Annals of medicine, 53(1), } \\
\text { 34-42. }\end{array}$} & \multirow{4}{*}{$\begin{array}{l}\text { Table } \\
\text { S1 }\end{array}$} \\
\hline & 0.7780 & 0.9500 & 0.9690 & 0.6780 & -0.0001 & 0.9998 & 0.7760 & 0.9494 & 0.9693 & 0.6805 & & \\
\hline & 0.8520 & 0.9000 & 0.9450 & 0.7500 & -0.0001 & 0.9999 & 0.8514 & 0.8995 & 0.9453 & 0.7510 & & \\
\hline & 0.9510 & 0.7500 & 0.8850 & 0.8850 & 0.0002 & 1.0003 & 0.9518 & 0.7532 & 0.8833 & 0.8833 & & \\
\hline \multirow[t]{4}{*}{31} & 0.7590 & 0.8640 & 0.2310 & 0.9850 & -0.0001 & 0.9992 & 0.7564 & 0.8623 & 0.2335 & 0.9852 & \multirow{4}{*}{$\begin{array}{l}\text { Spangler D, Blomberg H Smekal D. (2021). Prehospital } \\
\text { identification of Covid-19: an observational study. Scandinavian } \\
\text { Journal of Trauma, Resuscitation and Emergency Medicine, } \\
\text { 29(1), 1-10. }\end{array}$} & \multirow[t]{4}{*}{ Table 3} \\
\hline & 0.8220 & 0.7820 & 0.1690 & 0.9880 & 0.0001 & 1.0007 & 0.8236 & 0.7838 & 0.1675 & 0.9879 & & \\
\hline & 0.7110 & 0.7300 & 0.2470 & 0.9530 & 0.0000 & 1.0000 & 0.7110 & 0.7300 & 0.2470 & 0.9530 & & \\
\hline & 0.7910 & 0.5440 & 0.1770 & 0.9540 & -0.0002 & 0.9965 & 0.7890 & 0.5410 & 0.1788 & 0.9545 & & \\
\hline \multirow[t]{2}{*}{32} & 1.0000 & 0.0400 & 0.3900 & 1.0000 & 0.0000 & 1.0000 & 1.0000 & \#DIV/0! & \#DIV/0! & 1.0000 & \multirow{2}{*}{$\begin{array}{l}\text { Akhavan AR, Habboushe JP, Gulati R, Iheagwara O, Watterson J, } \\
\text { Thomas S, Swartz JL, Koziatek CA, Lee DC. Risk Stratification of }\end{array}$} & \multirow[t]{2}{*}{ Table 3} \\
\hline & 0.9800 & 0.1500 & 0.4100 & 0.9400 & 0.0013 & 1.0269 & 0.9840 & 0.1818 & 0.3556 & 0.9256 & & \\
\hline
\end{tabular}


Rushdi and Serag; IJPR, 6(2): 28-49, 2021; Article no.IJPR.65260

\begin{tabular}{|c|c|c|c|c|c|c|c|c|c|c|c|c|}
\hline \multirow[t]{20}{*}{ \# } & \multicolumn{4}{|c|}{ Original values } & \multicolumn{2}{|c|}{$\begin{array}{l}\text { Checking } \\
\text { Values }\end{array}$} & \multicolumn{4}{|c|}{ Computed values } & \multirow[t]{2}{*}{ Source } & \multirow{2}{*}{$\begin{array}{l}\text { Data } \\
\text { location } \\
\text { in } \\
\text { Source }\end{array}$} \\
\hline & Sens $_{i j}$ & Spec $_{i j}$ & $\mathbf{P P V}_{\mathrm{ij}}$ & $\mathrm{NPV}_{\mathrm{ij}}$ & $\mathrm{DCD}_{\mathrm{ij}}$ & $\mathbf{D C R}_{\mathrm{ij}}$ & Sens $_{i j}$ & Spec $_{i j}$ & $\mathbf{P P V}_{\mathrm{ij}}$ & $\mathrm{NPV}_{\mathrm{ij}}$ & & \\
\hline & 0.9700 & 0.1800 & 0.4200 & 0.9000 & -0.0008 & 0.9854 & 0.9674 & 0.1678 & 0.4409 & 0.9074 & \multirow{18}{*}{$\begin{array}{l}\text { COVID-19 Patients Using Ambulatory Oxygen Saturation in the } \\
\text { Emergency Department. Western Journal of Emergency } \\
\text { Medicine. 2020;21(6):5-14. }\end{array}$} & \\
\hline & 0.9200 & 0.2900 & 0.4400 & 0.8600 & 0.0006 & 1.0072 & 0.9220 & 0.2956 & 0.4333 & 0.8567 & & \\
\hline & 0.8900 & 0.3600 & 0.4500 & 0.8400 & -0.0016 & 0.9832 & 0.8842 & 0.3468 & 0.4643 & 0.8476 & & \\
\hline & 0.8100 & 0.4300 & 0.4600 & 0.7900 & -0.0001 & 0.9984 & 0.8094 & 0.4291 & 0.4609 & 0.7906 & & \\
\hline & 0.7300 & 0.5000 & 0.4700 & 0.7500 & -0.0008 & 0.9904 & 0.7268 & 0.4960 & 0.4740 & 0.7530 & & \\
\hline & 0.6000 & 0.5900 & 0.4700 & 0.7100 & 0.0003 & 1.0050 & 0.6014 & 0.5914 & 0.4686 & 0.7088 & & \\
\hline & 0.3500 & 0.8000 & 0.5100 & 0.6700 & -0.0009 & 0.9833 & 0.3457 & 0.7969 & 0.5148 & 0.6742 & & \\
\hline & 0.3400 & 0.8500 & 0.5800 & 0.6800 & 0.0002 & 1.0027 & 0.3412 & 0.8507 & 0.5787 & 0.6789 & & \\
\hline & 0.9800 & 0.0500 & 0.1000 & 0.9600 & 0.0001 & 1.0208 & 0.9806 & 0.0516 & 0.0970 & 0.9587 & & \\
\hline & 0.9800 & 0.1100 & 0.1000 & 0.9800 & -0.0002 & 0.9778 & 0.9778 & 0.1000 & 0.1100 & 0.9820 & & \\
\hline & 0.9000 & 0.2300 & 0.1100 & 0.9500 & -0.0012 & 0.9142 & 0.8872 & 0.2069 & 0.1240 & 0.9560 & & \\
\hline & 0.8200 & 0.4200 & 0.1300 & 0.9600 & 0.0010 & 1.0349 & 0.8320 & 0.4405 & 0.1208 & 0.9567 & & \\
\hline & 0.7400 & 0.5800 & 0.1600 & 0.9500 & -0.0014 & 0.9706 & 0.7238 & 0.5598 & 0.1714 & 0.9538 & & \\
\hline & 0.5400 & 0.7300 & 0.1800 & 0.9400 & 0.0016 & 1.0355 & 0.5599 & 0.7455 & 0.1685 & 0.9353 & & \\
\hline & 0.2800 & 0.8300 & 0.1500 & 0.9200 & 0.0011 & 1.0717 & 0.2936 & 0.8392 & 0.1417 & 0.9150 & & \\
\hline & 0.2000 & 0.9000 & 0.1700 & 0.9100 & -0.0011 & 0.9308 & 0.1871 & 0.8923 & 0.1820 & 0.9166 & & \\
\hline & 0.0800 & 0.9600 & 0.1600 & 0.9100 & -0.0004 & 0.9231 & 0.0743 & 0.9568 & 0.1711 & 0.9164 & & \\
\hline & 0.0200 & 0.9800 & 0.1000 & 0.9000 & 0.0000 & \#DIV/0! & 0.0200 & 0.9800 & 0.1000 & 0.9000 & & \\
\hline \multirow[t]{5}{*}{33} & 0.9890 & 0.2070 & 0.4830 & 0.9600 & -0.0002 & 0.9979 & 0.9885 & 0.1996 & 0.4944 & 0.9617 & \multirow{5}{*}{$\begin{array}{l}\text { Sung J, Choudry N, Bachour R. Development and validation of a } \\
\text { simple risk score for diagnosing COVID-19 in the emergency } \\
\text { room. Epidemiology \& Infection. 2020;148(e273):1-7. }\end{array}$} & \multirow[t]{5}{*}{ Table 5} \\
\hline & 0.9770 & 0.4740 & 0.5820 & 0.9650 & 0.0000 & 1.0001 & 0.9771 & 0.4747 & 0.5813 & 0.9649 & & \\
\hline & 0.9800 & 0.1630 & 0.4000 & 0.9330 & -0.0002 & 0.9967 & 0.9795 & 0.1593 & 0.4066 & 0.9347 & & \\
\hline & 0.2760 & 0.9830 & 0.9230 & 0.6440 & -0.0001 & 0.9992 & 0.2727 & 0.9827 & 0.9242 & 0.6478 & & \\
\hline & 0.2860 & 0.9770 & 0.8750 & 0.7060 & -0.0001 & 0.9992 & 0.2835 & 0.9767 & 0.8763 & 0.7085 & & \\
\hline \multirow[t]{7}{*}{34} & 0.9630 & 0.6530 & 0.1310 & 0.9970 & 0.0000 & 1.0005 & 0.9638 & 0.6581 & 0.1284 & 0.9969 & \multirow{7}{*}{$\begin{array}{l}\text { Dagan N, Barda N, Riesel D, Grotto I, Sadetzki S \& Balicer R. } \\
\text { (2020). A score-based risk model for predicting severe COVID-19 } \\
\text { infection as a key component of lockdown exit strategy. medRxiv. } \\
\text { Available at: } \\
\text { https://www.medrxiv.org/content/medrxiv/early/2020/05/23/2020.0 } \\
5.20 .20108571 . \text { full.pdf. }\end{array}$} & \multirow{7}{*}{ Table 2} \\
\hline & 0.9190 & 0.7950 & 0.1960 & 0.9940 & -0.0003 & 0.9979 & 0.9124 & 0.7807 & 0.2099 & 0.9945 & & \\
\hline & 0.4300 & 0.9520 & 0.3260 & 0.9690 & 0.0001 & 1.0007 & 0.4326 & 0.9525 & 0.3237 & 0.9687 & & \\
\hline & 0.4890 & 0.8430 & 0.1450 & 0.9680 & 0.0000 & 0.9996 & 0.4886 & 0.8428 & 0.1452 & 0.9680 & & \\
\hline & 0.0810 & 0.2050 & 0.0600 & 0.8040 & 0.0322 & 0.0656 & 0.5038 & 0.7482 & 0.0055 & 0.2626 & & \\
\hline & 0.9630 & 0.5750 & 0.1360 & 0.9960 & 0.0002 & 1.0030 & 0.9666 & 0.6009 & 0.1239 & 0.9955 & & \\
\hline & 0.9190 & 0.7380 & 0.1960 & 0.9920 & -0.0002 & 0.9982 & 0.9148 & 0.7271 & 0.2049 & 0.9924 & & \\
\hline
\end{tabular}


Rushdi and Serag; IJPR, 6(2): 28-49, 2021; Article no.IJPR. 65260

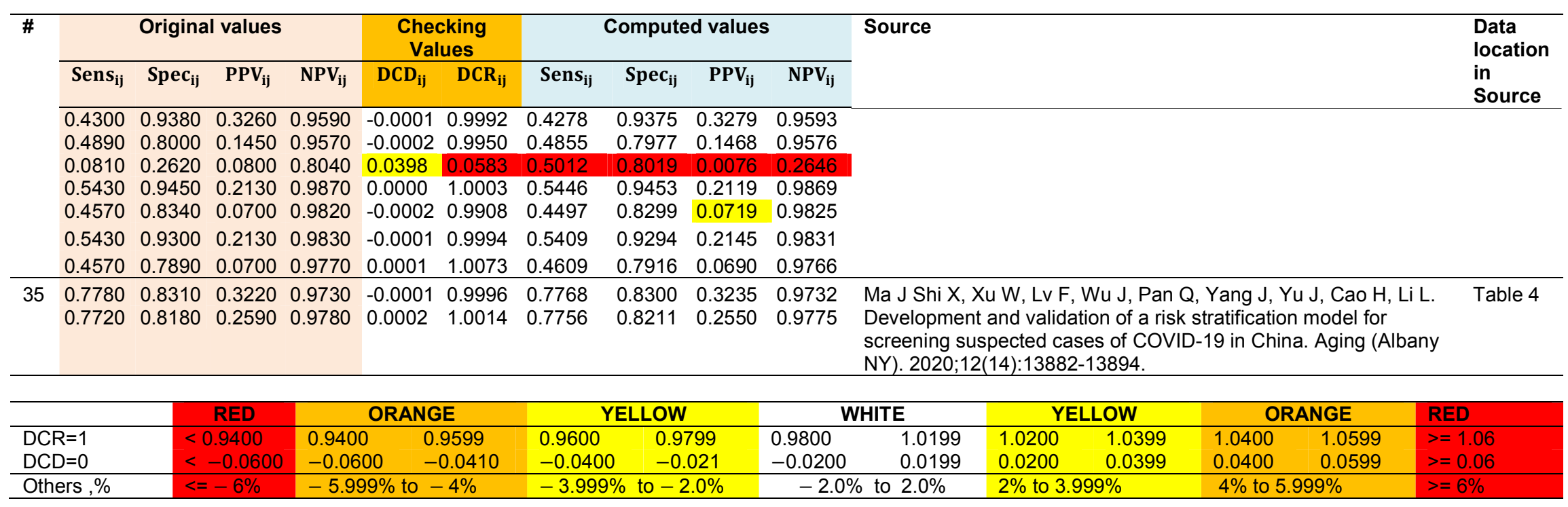




\section{CONCLUSIONS}

We provide a novel method for validating any purported set of the four most prominent indicators of diagnostic testing (Sensitivity, Specificity, Positive Predictive Value, and Negative Predictive Value), by observing that these indicators constitute three rather than four independent quantities. This observation has virtually been unheard of in the open medical literature. Up to date, researchers who were unaware of this observation could possibly be somewhat excused, but, from now on, this observation should be popularized and respected. We defined two functions that check consistency for any set of four numerical values claimed to be the four basic diagnostic indicators, and naturally belonging to the interval $[0.0,1.0]$. Most of data we came across herein and earlier in $[17,45]$ met our criterion for consistency, but in a few cases, there were obvious blunders. The percentage of inconsistent cases and the severity of deviations therein seem definitely higher for the present COVID-19 data than they were in $[17,45]$. Our preliminary results seem to strengthen our suspicions of compromising the quality of publications in the COVID-19 era.

Our study has a few limitations that allow for possible improvements in future work. Our validating formulas express any one of the four diagnostic indicators in terms of the other three, only under the assumptions that each of the four indicators exists, and that no division by zero is encountered. Though our validating formulas are correct for any combination of input values, they occasionally produce the indefinite value $(0 / 0)$, which need to be defined through a limit operation [4]. The threshold for accepting or rejecting purported sets is selected in an arbitrary (albeit plausible) way. Such a threshold might be chosen in other (statistically-rigorous) ways. Another major limitation of our work is that our coverage of publications in the COVID-19 era is far from being exhaustive. We did not set up an organized plan to cover all publications dealing with COVID-19, or to select a representative sample of these publications. We simply presented an adequate number of publications that we came across, and that we could access, in the Google Scholar database, in which we could locate a complete set of the four diagnostic indicators reported on COVID-19. We honestly report the results of all these publications that we have seen, with no exclusion of any of them. In summary, we suggest that some further literature-encompassing statistically- rigorous investigation of the pandemic-induced quality deterioration should be pursued.

\section{COMPETING INTERESTS}

The authors have declared that no competing interests exist.

\section{REFERENCES}

1. Del Rio C, Collins LF, Malani P. Long-term health consequences of COVID-19. The Journal of the American Medical Association (JAMA). 2020;324(17):17231724.

2. Pak A, Adegboye OA, Adekunle Al, Rahman KM, McBryde ES, Eisen DP. Economic consequences of the COVID-19 outbreak: The need for epidemic preparedness. Frontiers in Public Health. 2020;8(241):1-4.

3. Ritchie $\mathrm{K}$, Chan D, Watermeyer T. The cognitive consequences of the COVID-19 epidemic: collateral damage? Brain Communications. 2020;2(2):fcaa069,1-5.

4. Rushdi AMA, Serag HA. Solutions of ternary problems of conditional probability with applications to mathematical epidemiology and the COVID-19 pandemic, International Journal of Mathematical, Engineering and Management Sciences. 2020;5(5):787-811.

5. Rushdi MA, Rushdi AM. Modeling virus spread rate via modern techniques of dimensional analysis. Journal of King Abdulaziz University: Computing and Information Technology Sciences. 2020; 9(2):47-66.

6. Rushdi MA, Rushdi AM. Modeling coronavirus spread rate utilizing dimensional analysis via an irredundant set of fundamental quantities. International Journal of Pathogen Research. 2020; 5(3):8-21.

7. Shin MD, Shukla S, Chung YH, Beiss V, Chan SK, Ortega-Rivera OA et al. COVID19 vaccine development and a potential nanomaterial path forward. Nature Nanotechnology. 2020;15(8):646-455.

8. Dinis-Oliveira RJ. COVID-19 research: Pandemic versus "paperdemic", integrity, values and risks of the "speed science". Forensic Sciences Research. 2020;5(2): 174-187.

9. Bramstedt KA. The carnage of substandard research during the COVID-19 pandemic: A call for quality. Journal of Medical Ethics. 2020;46(12):803-807. 
10. Qaiser D. COVID-19 Pandemic and Research Publications; Necessity of Maintaining Scientific Integrity. International Annals of Science. 2020;10(1):1-6.

11. Silverberg SL, Puchalski Ritchie LM, Gobat $\mathrm{N}$, Nichol A, Murthy S. Clinicianresearcher's perspectives on clinical research during the COVID19 pandemic. Plos one. 2020;15(12):e0243525.

12. Daube D, Ruhrmann G. Einführung zur Qualität von Medizinjournalismus und erste Einschätzungen zur COVID-19Berichterstattung (Introduction to the quality of Medical Journalism and Initial Assessments of COVID-19 Media Coverage), BundesgesundheitsblattGesundheitsforschung-Gesundheitsschutz. 2020;1-9.

13. Boschiero MN, Carvalho TA, de Lima Marson FA. Retraction in the era of COVID19 and its influence on evidence-based medicine: Is science in jeopardy? Pulmonology; 2020.

14. Ferreira JP, Epstein M, Zannad F. The decline of the experimental paradigm during the COVID-19 pandemic: A template for the future. The American Journal of Medicine; 2020.

15. Campbell M, Is Research Quality Being Compromised During the COVID-19 Pandemic? Posted; 2020.

Available:https://www.technologynetworks.c om/tn/articles/is-research-quality-beingcompromised-during-the-covid-19pandemic-333849

16. London AJ, Kimmelman J. Against pandemic research exceptionalism. Science. 2020;368(6490):476-477.

17. Serag HA, Rushdi AMA. Checking consistency among the four basic indicators of diagnostic testing in Saudi medical journals, Asian Journal of Medical Principles and Clinical Practice. 2021;4(1): 14-27.

18. Da Silva JA, Bornemann-Cimenti $\mathrm{H}$, Tsigaris $\mathrm{P}$. Optimizing peer review to minimize the risk of retracting COVID-19related literature. Medicine, Health Care and Philosophy. Published. 2020;1-6.

19. Vlasschaert C, Topf J, Hiremath S. Proliferation of papers and preprints during the COVID-19 pandemic: Progress or problems with peer review? Advances in Chronic Kidney Disease. 2020;27(5):418426.

20. Horbach SP. No time for that now! Qualitative changes in manuscript peer review during the Covid-19 pandemic. Research Evaluation. 2021;rvaa037:1-9.

21. Steen RG. Retractions in the scientific literature: is the incidence of research fraud increasing? Journal of Medical Ethics. 2011;37(4):249-53.

22. Vuong Q-H. Reform retractions to make them more transparent. Nature. 2020; 582(7811):149.

23. Vuong Q-H. The limitations of retraction notices and the heroic acts of authors who correct the scholarly record: An analysis of retractions of papers published from 1975 to 2019. Learned Publishing. 2020;33(2): 119-130.

24. Yeo-Teh NS, Tang BL. An alarming retraction rate for scientific publications on Coronavirus Disease 2019 (COVID-19). Accountability in Research. 2021;28(1):4753.

25. Abritis A, Marcus A, Oransky I. An alarming and exceptionally high rate of COVID-19 retractions? Accountability in Research. 2020;28(1):58-59.

26. Soltani P, Patini R. Retracted COVID-19 articles: A side-effect of the hot race to publication. Scientometrics. 2020;125(1): 819-22.

27. Buyse M, George SL, Evans S, Geller NL, Ranstam J, Scherrer B et al. The role of biostatistics in the prevention, detection and treatment of fraud in clinical trials. Statistics in Medicine. 1999;18(24):343551.

28. Fanelli D. How many scientists fabricate and falsify research? A systematic review and meta-analysis of survey data. PloS one. 2009 May 29;4(5):e5738.

29. Simonsohn U. Just post it: The lesson from two cases of fabricated data detected by statistics alone. Psychological Science. 2013;24(10):1875-88.

30. Pollach G, Brunkhorst F, Mipando M, Namboya F, Mndolo S, Luiz T. The first digit law-A hypothesis on its possible impact on medicine and development aid. Medical Hypotheses. 2016;97:102-106.

31. Hartgerink C, Wicherts J, Van Assen M. The value of statistical tools to detect data fabrication. Research Ideas and Outcomes. 2016;2:e8860.

DOI: https://doi.org/10.3897/rio.2.e8860

32. Wicherts JM. The weak spots in contemporary science (and how to fix them). Animals. 2017;7(12):90. Available:https://doi.org/10.3390/ani71200 90 
33. Piraino SW. Issues in the statistical detection of data fabrication and data errors in the scientific literature: Simulation study and reanalysis of Carlisle. Bio Rxiv. 2017;179135.

34. Mascha EJ, Vetter TR, Pittet JF. An appraisal of the Carlisle-Stouffer-Fisher method for assessing study data integrity and fraud. Anesthesia and Analgesia. 2017; 125(4):1381-1385.

35. Hüllemann $S$, Schüpfer $G$, Mauch J. Application of Benford's law: A valuable tool for detecting scientific papers with fabricated data? Der Anaesthesist. 2017; 66(10):795-802.

36. Hartgerink $\mathrm{CH}$, Voelkel JG, Wicherts J, Van Assen MA. Detection of data fabrication using statistical tools; 2019. Acailable:https://psyarxiv.com/jkws4/

37. Montoya JM. Benford's Law with small sample sizes: A new exact test useful in health sciences during epidemics, Revista Salud Uis. 2020;52(2):161-163.

38. Rushdi RA, Rushdi AM. Common fallacies of probability in medical context: A simple mathematical exposition, Journal of Advances in Medicine and Medical Research. 2018;26(1):1-21.

39. Rushdi RAM, Rushdi AMA. Mathematics and examples for avoiding common probability fallacies in Medical Disciplines. Chapter 11 in Current Trends in Medicine and Medical Research, Book Publishers International, Hooghly, West Bengal, India. 2019;1:106-132.

40. Rushdi RA, Rushdi AM. Karnaugh-map utility in medical studies: The case of Fetal Malnutrition. International Journal of Mathematical, Engineering and Management Sciences. 2018;3(3):220244.

41. Rushdi AM, Badawi RMS. Karnaugh-map utilization in Boolean analysis: The case of war termination. Journal of Qassim University: Engineering and Computer Sciences. 2017;10(1):53-88.

42. Rushdi AM, Badawi RMS. Karnaugh map utilization in coincidence analysis. Journal of King Abdulaziz University: Computing and Information Technology Sciences. 2017;6(1-2):37-44.

43. Rushdi AMA. Utilization of Karnaugh maps in multi-value qualitative comparative analysis, International Journal of Mathematical, Engineering and Management Sciences (IJMEMS). 2018; 3(1):28-46.
44. Rushdi AMA, Badawi RMS. Computer engineers look at qualitative comparative analysis. International Journal of Mathematical, Engineering and Management Sciences (IJMEMS). 2019; 4(4):851-860.

45. Rushdi AMA, Serag HA. Inter-relationships among the four basic measures of diagnostic testing: A signal-flow-graph approach, Journal of King Abdulaziz University: Computing and Information Technology Sciences. 2021;10(1).

46. Rushdi RA, Rushdi AM, Talmees FA. Novel pedagogical methods for conditional-probability computations in medical disciplines, Journal of Advances in Medicine and Medical Research. 2018; 25(10):1-15.

47. Rushdi AMA, Talmees FA. An exposition of the eight basic measures in diagnostic testing using several pedagogical tools, Journal of Advances in Mathematics and Computer Science. 2018;26(3): $1-17$.

48. Rushdi AMA, Talmees FA. Computations of the Eight Basic Measures in Diagnostic Testing. Chapter 6 in Advances in Mathematics and Computer Science, Book Publishers International, Hooghly, West Bengal, India. 2019:2;66-87.

49. Rushdi RA, Rushdi AM, Talmees FA. Review of Methods for ConditionalProbability computations in Medical Disciplines, Chapter 7 in highlights on Medicine and Medical Research, Book Publishers International, Hooghly, West Bengal, India. 2021;76-94.

50. Canbek G, Sagiroglu S, Temizel TT, Baykal N. Binary classification performance measures/metrics: A comprehensive visualized roadmap to gain new insights. In 2017 International IEEE Conference on Computer Science and Engineering (UBMK). 2017;821-826.

51. Rushdi AM. Reliability of k-out-of-n Systems. Chapter 5 in Misra KB, New trends in system reliability evaluation, fundamental studies in engineering, Elsevier Science Publishers, Amsterdam, The Netherlands. 1993;16:185-227.

52. Rushdi AM, Rushdi MA, Switchingalgebraic analysis of system reliability. Chapter 6 in Ram MM, Davim P (Editors). Advances in Reliability and System Engineering. Springer International Publishing, Cham, Switzerland. 2017;139161. 
53. Rushdi AM, Rushdi MA. Mathematics and examples of the modern syllogistic method of propositional logic. Mathematics Applied in Information Systems, Bentham Science Publishers, Emirate of Sharjah, United Arab Emirates. 2018;6:123-167.

54. Rushdi AM, Ghaleb FA. On self-inverse binary matrices over the binary Galois field. Journal of Mathematics and Statistics. 2013;9(3):238-248.

55. Rushdi AM, Ghaleb FA. A tutorial exposition of semi-tensor products of matrices with a stress on their representation of Boolean functions. Journal of King Abdulaziz University: Computing and Information Technology Sciences. 2016;5(1):3-30.

56. Rushdi AM, Alsogati AA. The eigenvectors of the transition matrix as predictors of the dynamics of a synchronous Boolean network. Journal of Advances in Mathematics and Computer Science. 2020;35(6):80-99.

57. Goldberg D. What every computer scientist should know about floating-point arithmetic. ACM Computing Surveys (CSUR). 1991;23(1):5-48.

58. Luo JC. Analysis of round-off errors. Applied Mathematics Letters. 1992;5(4): 77-80.

59. Higham NJ. The accuracy of floating point summation. SIAM Journal on Scientific Computing. 1993;14(4):783-799.

60. Rushdi AM, Al-Hindi KA. A table for the lower boundary of the region of useful redundancy for k-out-of-n systems. Microelectronics and Reliability. 1993;33(7): 979-992.

61. Bailey $\mathrm{DH}$. High-precision floating-point arithmetic in scientific computation. Computing in Science and Engineering. 2005;7(3):54-61.

62. Al-Qasimi AM, Rushdi AM. A tutorial on how to efficiently calculate and format tables of the binomial distribution. Journal of King Abdulaziz University: Engineering Sciences. 2008;19(1):3-18.

63. Fowkes FG, Fulton P. Critical appraisal of published research: Introductory guidelines. BMJ: British Medical Journal. 1991; 302(6785):1136-1140.

64. Katrak P, Bialocerkowski AE, MassyWestropp N, Kumar VS, Grimmer KA. A systematic review of the content of critical appraisal tools. BMC Medical Research Methodology. 2004;4(1):1-11.

65. Young JM, Solomon MJ. How to critically appraise an article. Nature Clinical Practice Gastroenterology and Hepatology. 2009; 6(2):82-91.

66. Crowe M, Sheppard L. A review of critical appraisal tools show they lack rigor: Alternative tool structure is proposed. Journal of Clinical Epidemiology. 2011; 64(1):79-89.

67. Moher D, Altman DG. Four proposals to help improve the medical research literature. PLoS Med. 2015;12(9): e1001864.

68. Moher D, Glasziou P, Chalmers I, Nasser $M$, Bossuyt PM, Korevaar DA, et al. Increasing value and reducing waste in biomedical research: Who's listening? The Lancet. 2016;387(10027):1573-1586.

69. Johansen M, Thomsen SF. Guidelines for reporting medical research: A critical appraisal. International Scholarly Research Notices. 2016;2016:1-7.

70. Rahmani MR, Ayoobi F, Assar S, Jalalpour S, Rezaeian M. معرفى دستور العمل ارتقاء

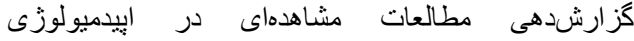
(Introducing guidelines for improving the reporting of observational studies in epidemiology). Journal of Rafsanjan University of Medical Sciences. In Persian (Farsi). 2017;16(3):257-274.

71. Grech V. Write a Scientific Paper (WASP): Guidelines for reporting medical research. Early Human Development. 2019;134:5557.

(C) 2021 Rushdi and Serag; This is an Open Access article distributed under the terms of the Creative Commons Attribution License (http://creativecommons.org/licenses/by/4.0), which permits unrestricted use, distribution, and reproduction in any medium, provided the original work is properly cited.

Peer-review history:

The peer review history for this paper can be accessed here: http://www.sdiarticle4.com/review-history/65260 